\title{
Dynamic BMP signaling polarized by Toll patterns the dorsoventral axis in a hemimetabolous insect
}

\author{
Lena Sachs ${ }^{1 \dagger}$, Yen-Ta Chen ${ }^{1+}$, Axel Drechsler ${ }^{1,2}$, Jeremy A Lynch ${ }^{1,3}$, \\ Kristen A Panfilio', Michael Lässig ${ }^{4}$, Johannes Berg ${ }^{4}$, Siegfried Roth ${ }^{1 *}$ \\ ${ }^{1}$ Institute for Developmental Biology, University of Cologne, Köln, Germany; \\ ${ }^{2}$ Bundesministerium für Umwelt, Naturschutz, Bau und Reaktorsicherheit, Bonn, \\ Germany; ${ }^{3}$ Department of Biological Sciences, University of Illinois at Chicago, \\ Chicago, United States; ${ }^{4}$ Institute for Theoretical Physics, University of Cologne, \\ Cologne, Germany
}

*For correspondence: siegfried. roth@uni-koeln.de

TThese authors contributed equally to this work

Competing interests: The authors declare that no competing interests exist.

Funding: See page 22

Received: 05 November 2014 Accepted: 12 April 2015 Published: 12 May 2015

Reviewing editor: Naama Barkai, Weizmann Institute of Science, Israel

(c) Copyright Sachs et al. This article is distributed under the terms of the Creative Commons Attribution License, which permits unrestricted use and redistribution provided that the original author and source are credited.

\begin{abstract}
Toll-dependent patterning of the dorsoventral axis in Drosophila represents one of the best understood gene regulatory networks. However, its evolutionary origin has remained elusive. Outside the insects Toll is not known for a patterning function, but rather for a role in pathogen defense. Here, we show that in the milkweed bug Oncopeltus fasciatus, whose lineage split from Drosophila's more than 350 million years ago, Toll is only required to polarize a dynamic BMP signaling network. A theoretical model reveals that this network has self-regulatory properties and that shallow Toll signaling gradients are sufficient to initiate axis formation. Such gradients can account for the experimentally observed twinning of insect embryos upon egg fragmentation and might have evolved from a state of uniform Toll activity associated with protecting insect eggs against pathogens.

DOI: 10.7554/eLife.05502.001
\end{abstract}

\section{Introduction}

In the fly Drosophila melanogaster, the Toll pathway has essential functions both for innate immunity and for dorsoventral (DV) axis formation (Leulier and Lemaitre, 2008; Stein and Stevens, 2014). While Toll's immune function is broadly conserved in animals ranging from hydra to humans, its role in axis formation appears to be an evolutionary novelty of insects (Leulier and Lemaitre, 2008; Franzenburg et al., 2012; Gilmore and Wolenski, 2012). Other animals do not employ Toll but rather use BMP signaling to establish their DV axis (De Robertis, 2008). BMP signaling also plays a crucial, but spatially restricted role in Drosophila DV patterning (O'Connor et al., 2006). This suggests that Toll signaling was recruited into an ancestral BMP-based patterning network during evolution of the insect lineage.

So far molecular studies of DV patterning in insects have been largely restricted to the most speciose supraorder, Holometabola, the insects with complete metamorphosis (Lynch and Roth, 2011). However, already within the Holometabola, a clear evolutionary trend was observed: the more basally branching lineages show an increased reliance on BMP signaling while the importance of Toll signaling is reduced (Figure 1). In Drosophila, both the polarity and pattern of the DV axis depend on a stable long range gradient of Toll signaling that promotes the graded nuclear uptake of the NF-kB transcription factor Dorsal (Reeves and Stathopoulos, 2009). NF-kB/Dorsal acts in a concentrationdependent manner to activate or repress genes required for DV cell fate specification (Figure 1). The ventral cell fates of the mesoderm, mesectoderm and neuroectoderm directly depend on NF-kB/ Dorsal target genes. The dorsal cell fates (non-neurogenic ectoderm and extraembryonic 
eLife digest How an animal develops from a fertilized egg has fascinated scientists for decades. As such, much effort has gone into answering the related question: what makes the belly (or underside) of an animal develop differently from its back?

Like almost all other biological processes, the development of an embryo is controlled by interactions between different molecules within cells and tissues. Some of these molecules promote the activity of others; some have the opposite effect; and together these molecules and their interactions form 'signaling networks'. One such network, which involves a protein called BMP, is needed to establish the belly-to-back axis of nearly all animals. However, insects are a unique exception. Most insects (including flies, beetles and wasps) use a different signaling network to control their development from their belly to their back, one that involves a protein called Toll instead. This is unexpected because, in other animals, Toll proteins are best known for their role in the immune system; and it remains unclear how Toll signaling came to be involved in insect development.

Now, Sachs, Chen et al. have studied an insect-called the milkweed bug-that is unlike most insects in that it does not have a larval stage (i.e., a maggot or a caterpillar) in its life-cycle. This characteristic makes the milkweed bug more similar to the ancestor of all insects, and thus makes it an excellent model to study how the Toll protein took over from BMP in insect development.

First, Sachs, Chen et al. experimentally reduced BMP signaling in milkweed bug embryos. This caused the embryos to develop features all around their bodies that are normally only associated with the animal's underside. In other insects, the development of these so-called 'ventral' features is typically controlled by Toll signaling; but in the milkweed bug this activity instead depends on a protein called Sog. Indeed, when Sachs, Chen et al. experimentally reduced both BMP and Toll signaling, the effect was the same as having reduced only BMP signaling, implying that Toll is not needed. Instead, Toll increased the level of the Sog protein up to a particular threshold. Above this threshold, Sog and BMP control each other to set out the animal's body plan. As insects evolved, it seems likely that Toll transitioned from being a trigger of BMP signaling to an important controller of insect development in its own right. But why was Toll put in the egg in the first place? It is possible that Toll was required to protect the eggs of early insects from attack by bacteria and fungi. Future work will now test this assumption and aim to explain how and why the Toll protein changed its role-from immunity to development-during evolution.

DOI: 10.7554/eLife.05502.002

amnioserosa) are determined in a more indirect way by Toll signaling restricting and polarizing an opposing BMP signaling gradient (O'Connor et al., 2006; Hong et al., 2008). The gene regulatory network (GNR) controlled by NF-kB/Dorsal has been extensively characterized. It encompasses 60-70 target genes which fall into six classes according to their enhancer structure (Hong et al., 2008). The sensitivity of these enhancers to different NF-kB/Dorsal concentrations is fine-tuned by ubiquitously distributed activators and repressors (Garcia and Stathopoulos, 2011; Ozdemir et al., 2014).

All major components of the BMP signaling network are controlled by NF-KB/Dorsal: one of the BMP ligands, the BMP2/4 homolog decapentaplegic (dpp), and the extracellular protease tolloid (t/d) are repressed by NF-KB/Dorsal and thus confined to the dorsal side of the embryo while an extracellular BMP inhibitor, the chordin homolog short gastrulation (sog), and a transcriptional repressor of BMP target genes (brinker, brk) are activated by NF-kB/Dorsal at the ventral side (Jazwinska et al., 1999; O'Connor et al., 2006; Hong et al., 2008; Rushlow and Shvartsman, 2012). The ventral-to-dorsal transport of Sog and Sog-BMP complexes and their dorsal cleavage by Tld leads to a BMP signaling gradient with peak levels at the dorsal side. Thus, Toll signaling via NF-kB/Dorsal not only provides precise spatial information for the ventral half of the axis, but indirectly also determines the patterning of the dorsal half. An independent maternal input at the dorsal side of Drosophila embryos has been discussed, but it apparently plays only a minor role (Araujo and Bier, 2000).

In contrast to Drosophila, Toll signaling in the beetle Tribolium castaneum is highly dynamic due to positive and negative feedback of Toll pathway components (Nunes da Fonseca et al., 2008). These dynamics lead to a temporally shifting NF-KB/Dorsal gradient which refines and disappears before the major DV patterning genes have established stable expression domains. This suggests that 


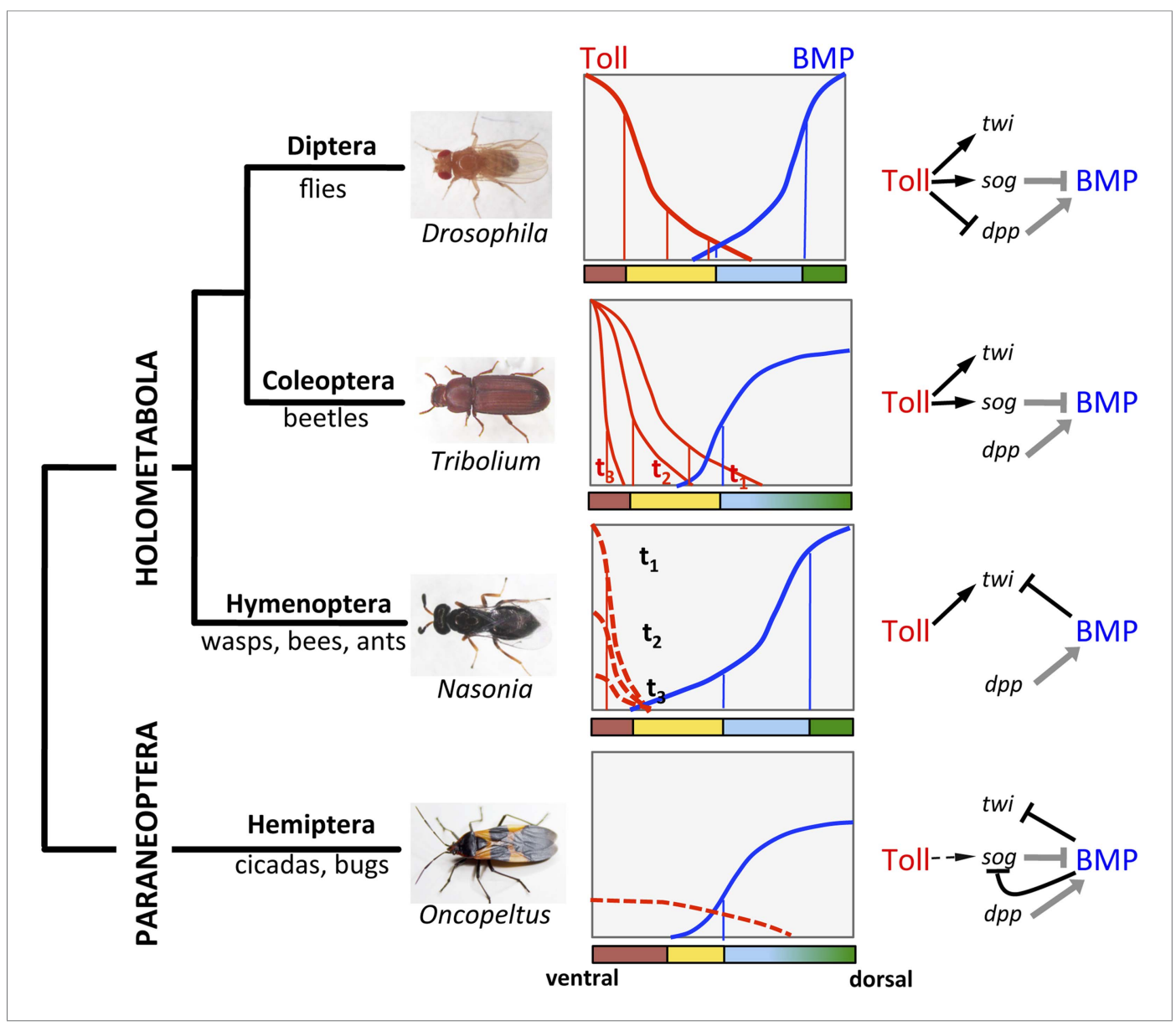

Figure 1. The evolution of Toll's role in dorsoventral (DV) patterning in insects. In holometabolous insects Toll signaling is activated by ventral eggshell cues and forms an activity gradient (red) that is essential at the very least for specifying the ventral-most cells on the DV axis, giving rise to the mesoderm (brown), by activating the gene twist (twi) (black arrow). In the fly Drosophila Toll signaling not only determines the mesoderm, but also the neuroectoderm (yellow) and restricts BMP signaling to the dorsal side through several parallel mechanisms, including the activation of the BMP inhibitor short gastrulation (sog) (black arrow) and repression of the major BMP ligand decapentaplegic (dpp) (black T-bar) (Hong et al., 2008; Reeves and Stathopoulos, 2009). On the dorsal side a BMP gradient (blue) is established (gray arrow and T-bar indicate BMP ligand production and inhibition, respectively) that specifies non-neurogenic ectoderm (blue) and extraembryonic tissue (green) (O'Connor et al., 2006). Toll signaling is dynamic in Tribolium and polarizes BMP signaling only by activating sog (Nunes da Fonseca et al., 2008). BMP signaling in turn has an increased role in ectodermal patterning compared to flies (van der Zee et al., 2006). In contrast to both Drosophila and Tribolium Toll signaling in the wasp Nasonia appears to be restricted to a narrow ventral region where it is only transiently active. Here, Toll signaling is required to induce mesodermal and mesectodermal fates. But the size of the mesodermal region as well as the fate and position of all other regions along the DV axis are determined by a BMP signaling gradient emanating from the dorsal side by an unknown (Toll-independent) mechanism (black T-bar indicates repression of twi) (Özüak et al., 2014a, 2014b). Thus, in the holometabolous insects BMP signaling gets increasingly more important towards basally branching groups, while Toll's role is diminished, but remains essential for ventral-most cell fates. Here we provide evidence that the bug Oncopeltus, representing the Hemiptera within the sister group of Holometabola (Paraneoptera), uses Toll signaling only as spatial cue (dashed black arrow) to polarize a dynamic BMP signaling network that establishes a gradient responsible for patterning the cell fates along the DV axis. The key regulatory element of this network is the transcriptional repression of sog by BMP signaling. A reaction-diffusion model which incorporates this regulatory element shows that the formation of stable BMP gradients requires only weakly polarized Toll signaling (Box 1). DOI: 10.7554/eLife.05502.003

NF-kB/Dorsal concentration thresholds play a less direct role in specifying these domains (Chen et al., 2000). In addition, NF- $\mathrm{kB} /$ Dorsal does not act as a repressor of BMP signaling components or as an activator of brk (Nunes da Fonseca et al., 2010). Consequently, the establishment of the BMP 
gradient entirely relies on ventral (NF-kB/Dorsal dependent) activation of sog. The BMP gradient in turn is required for all the polarity of the ectoderm (van der Zee et al., 2006). Thus, in Tribolium the direct role of Toll signaling is largely restricted to mesoderm and mesectoderm (Figure 1).

Finally, in the wasp Nasonia, representing the basal-most branch (Hymenoptera) of the Holometabola, Toll signaling appears to be active only in a narrow domain along the ventral midline, where it is required to induce ventral-most cell fates (Özüak et al., 2014b). However, the borders of the ventrally expressed genes are not defined by thresholds of Toll signaling, but rather by repressive BMP signaling. Thus, in Nasonia BMP signaling specifies gene expression domains along the entire DV axis (Figure 1). In this respect the DV system in Nasonia is similar to the ancestral type of DV axis formation in bilaterian animals. However, a closer look at the mechanisms of gradient formation reveals that the Nasonia system is highly derived even when compared to Drosophila. Functional studies show that the BMP gradient of Nasonia is established from a maternal source along the dorsal midline independent from ventral Toll signaling (Özüak et al., 2014b). Indeed, the Nasonia genome lacks a sog homolog and no ventrally expressed BMP inhibitor was identified (Özüak et al., 2014a). The establishment of BMP signaling gradients by an opposing inhibitor gradient of Chordin/Sog is however, one of the most conserved aspects of DV axis formation in Bilateria and is even preserved in flies (De Robertis, 2008). Moreover, given the fact that Nasonia also uses Toll for mesoderm/ mesectoderm induction, it establishes its DV axis in a bipolar manner employing independent signaling sources along the ventral and dorsal midline of the egg. Bipolar DV axis formation has so far not been described in any other system.

Despite all the variability found so far in Holometabola there are two common themes. (1) In more basal lineages BMP signaling is responsible for functions that are performed by Toll signaling in more derived lineages. (2) The ventral-most regions of the DV axis, giving rise to the mesoderm and mesectoderm, remain strictly dependent on Toll signaling. By studying a representative of insects with incomplete metamorphosis (Hemimetabola) we asked whether this situation is characteristic for all insects or whether a further reduction of the DV patterning function of Toll can be observed, allowing us to analyze how it originated.

To this end we investigated DV patterning in the milkweed bug, Oncopeltus fasciatus, representing the order Hemiptera, within the sister group (Paraneoptera) to the Holometabola (Liu and Kaufman, 2009). We provide evidence that in Oncopeltus Toll is indeed no longer essential for mesoderm formation since repression of BMP signaling suffices to induce mesoderm. Like in other systems inhibition of BMP signaling is accomplished by sog. However, the transcriptional regulation of sog in Oncopeltus is more dynamic than in the other well-studied systems. It combines uniform Tollindependent activation with ventral enhancement by Toll and repression by BMP. We build a theoretical model based on the experimental findings and show that the BMP/sog pathway in Oncopeltus exhibits self-organized patterning (Box 1). Specifically, the interplay of BMP-dependent sog repression and Sogdependent BMP transport generates a Turing instability (Turing, 1952). Toll's role in this system seems to be reduced to providing a trigger that enhances Sog activity above a certain threshold to initiate the patterning process. However, this patterning mechanism differs from the well-studied activator-inhibitor models (Gierer and Meinhardt, 1972); while sog is inhibited by BMP, there is no activator in our model.

\section{Results}

To mark different DV regions of Oncopeltus blastoderm embryos we chose twist (twi), a ventrally expressed marker for the mesoderm (Thisse et al., 1988) (Figure 2A lateral view), single minded (sim), a mesectodermal marker (Thomas et alo, 1988) expressed in lateral stripes bordering the mesoderm and in a ventral-anterior domain (Figure 2B lateral view), and short gastrulation (sog), the insect homolog of the BMP antagonist Chordin (Francois et al., 1994), which is expressed in a ventral domain slightly broader than that of twi (Figure $2 \mathrm{C}$ lateral view).

None of the known dorsally expressed genes from the Holometabola showed specific dorsal expression in early Oncopeltus embryos. This includes both the BMP signaling components (O'Connor et al., 2006) decapentalplegic (dpp), glass bottom boat (gbb), tolloid (tld) and twisted gastrulation (tsg) (Figure 2-figure supplement 1) as well as target genes potentially activated by BMP signaling like zerknüllt, pannier, dorsocross and iroquois (Panfilio et al., 2006; Nunes da Fonseca et al., 2010; Buchta et al., 2013) (data not shown). In the absence of dorsal marker genes we monitored the distribution of phosphorylated Mad (pMAD), the activated form of the transcription factor downstream of BMP signaling (Dorfman and Shilo, 2001; van der Zee et al., 2006). In early 


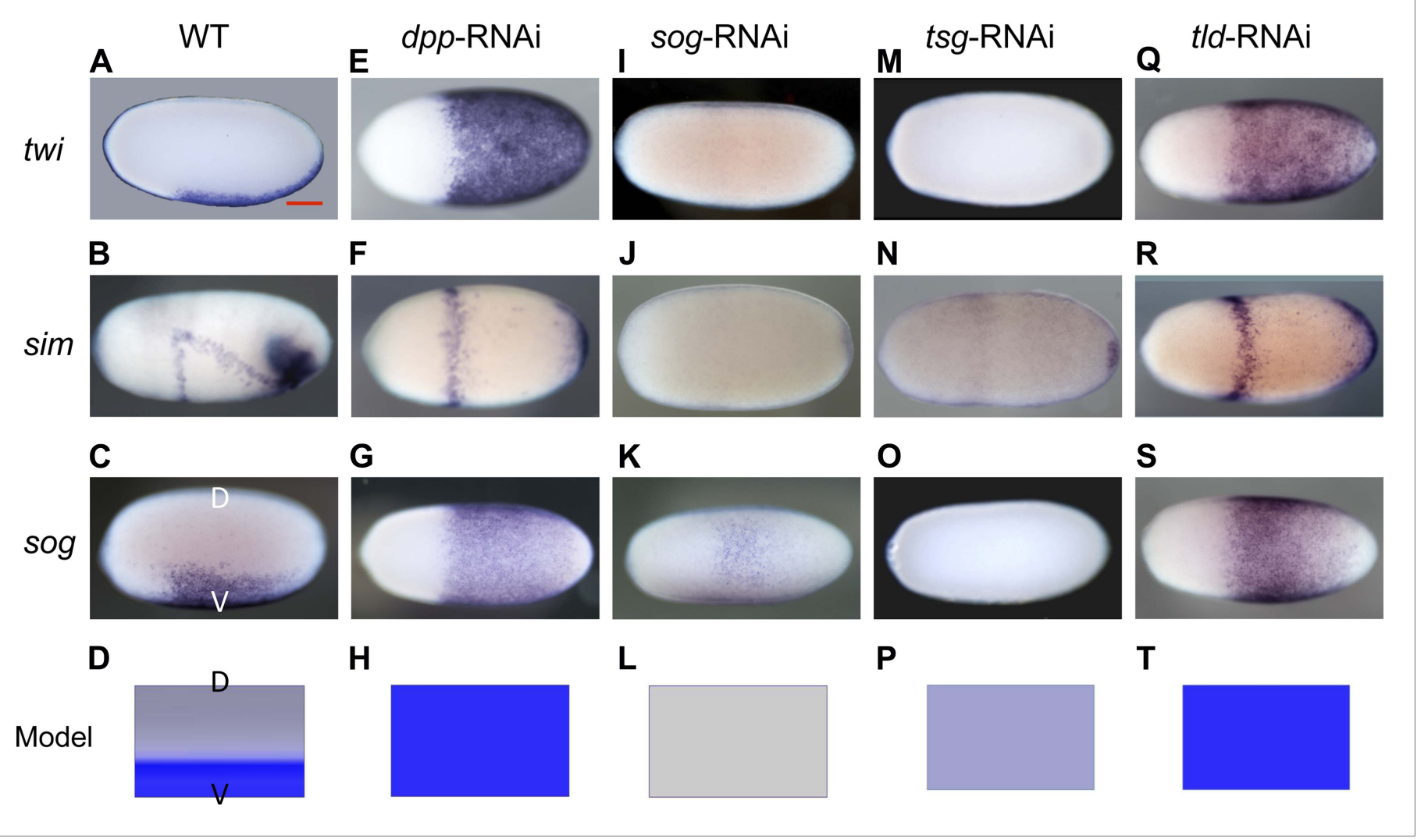

Figure 2. Knockdown (KD) of BMP signaling components results in completely ventralized (dpp-, tld-RNAi) or completely dorsalized (sog-, tsg-RNAi) embryos. Expression of twi (A, E, I, M, Q), $\operatorname{sim}(\mathbf{B}, \mathbf{F}, \mathbf{J}, \mathbf{N}, \mathbf{R})$ and $\operatorname{sog}(\mathbf{C}, \mathbf{G}, \mathbf{K}, \mathbf{O}, \mathbf{S})$ in wild type (wt) embryos (A-C), dpp-RNAi embryos (E-G), sog-RNAi

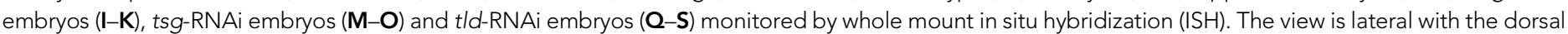
side pointing up $(\mathbf{A}-\mathbf{C})$, ventral $(\mathbf{K})$, or not determined as the expression is DV-symmetric (E-G, $\mathbf{I}, \mathbf{J}, \mathbf{M}-\mathbf{O}, \mathbf{Q}-\mathbf{S})$. Embryos are at the blastoderm stage ( 26-32 hpf: A, C, E-G, I-K, M, O, Q, S), or at the beginning of anatrepsis (posterior invagination of the embryo, $233-37$ hpf) (B). Scale bar (A) corresponds to $200 \mu \mathrm{m}$. For phenotype frequencies and confirmation of KD see Figure 2-figure supplement 2 and Figure 5-figure supplement 1. (D, H, L, P, T) Simulations of the reaction diffusion system described in Box 1 on a two-dimensional cylinder (Figure 10). Depicted is one half of the cylinder surface stretching from the dorsal (D) to the ventral ( $($ ) midline. Blue: sog expression $(\eta)$. Gray: BMP concentration (b). (D) In wt sog expression is confined to a ventral stripe. $(\mathbf{H})$ Loss of BMP $(b=0)$ leads to uniform derepression of sog. $(\mathbf{L})$ Loss of sog $(s=0)$ leads to uniformly high levels of BMP. (P) Loss of Tsg was modeled by assuming that no Sog-BMP complexes are formed $\left(k_{+}=0\right)$. This results in high BMP signaling throughout the embryo. (T) Loss of Tld was modeled by reducing the degradation constant of Sog $\left(\alpha_{s}\right)$ by $90 \%$. As Sog-BMP complexes are not degraded, BMP is not released, causing uniform derepression of sog. DOI: 10.7554/eLife.05502.004

The following figure supplements are available for figure 2:

Figure supplement 1. Expression of BMP signaling components during blastoderm.

DOI: 10.7554/eLife.05502.005

Figure supplement 2. Phenotype frequencies after parental RNAi.

DOI: 10.7554/eLife.05502.006

embryos pMAD accumulates more strongly in nuclei of dorsal than of ventral cells (Figure $3 A, F$; nuclear density can be used to distinguish dorsal and ventral regions of the embryos, Figure 3-figure supplement 1). Over time this asymmetry is enhanced. At the beginning of gastrulation, high levels of PMAD are restricted to the dorsal $30 \%$ of the egg circumference with sharp lateral borders (Figure 3-figure supplement 1). Within the domain of high nuclear concentrations the PMAD distribution is flat; i.e., it lacks the sharp peak along the dorsal midline which has been observed in Drosophila and Nasonia and is also less graded than the pMAD profile of Tribolium (Dorfman and Shilo, 2001; van der Zee et al., 2006; Özüak et al., 2014b).

Having established these four markers of distinct DV domains, we first analyzed the role of BMP signaling in Oncopeltus. Nuclear pMAD accumulation was largely abolished by knockdown (KD), via parental RNAi targeting the ortholog of Drosophila BMP ligand dpp (O'Connor et alo, 2006). This 


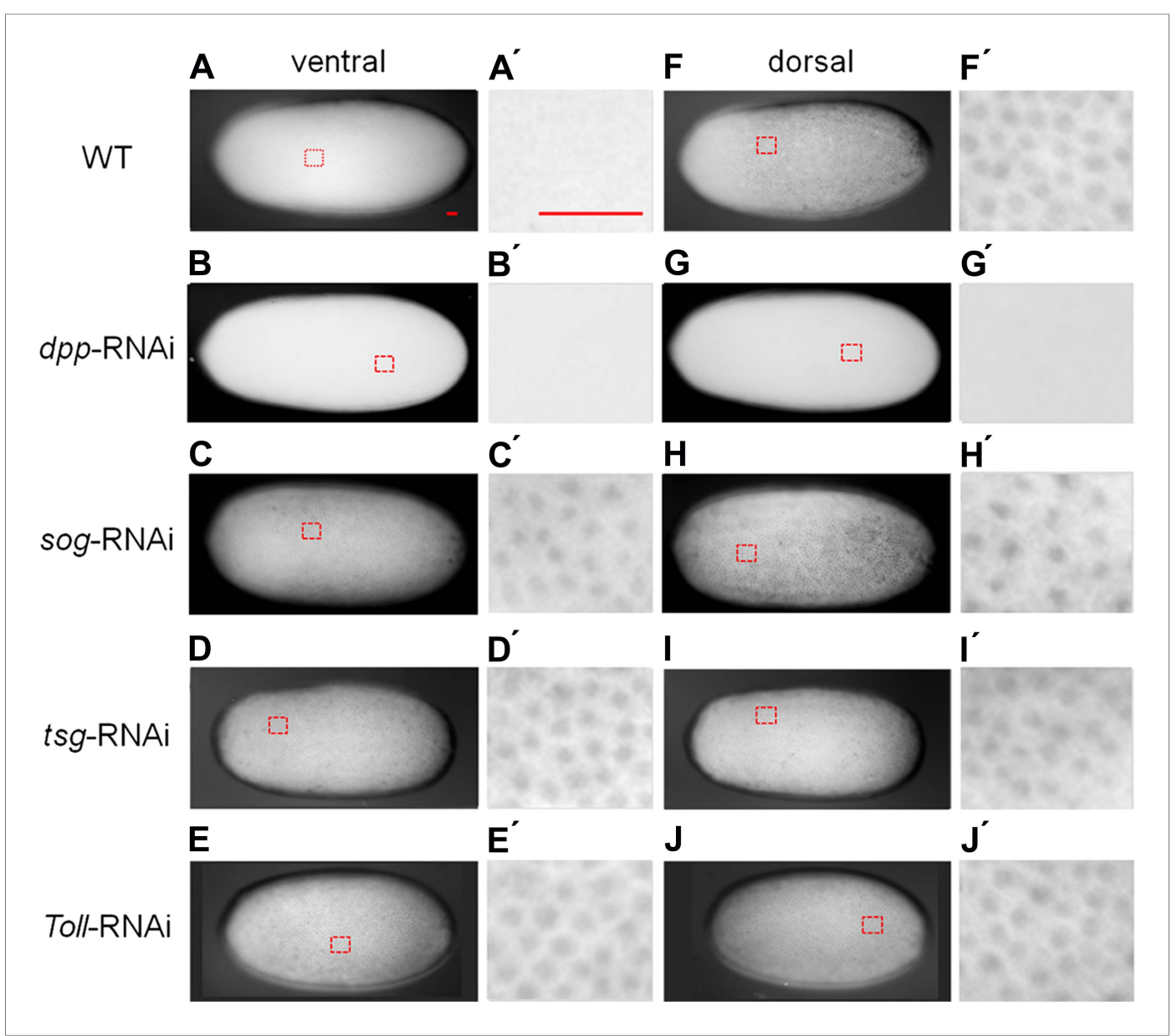

Figure 3. BMP signaling activity is uniformly abolished or expanded in ventralized or dorsalized phenotypes, respectively. pMAD distribution in blastoderm stage (26-32 hr post fertilization, hpf) wt (A, $\left.\mathbf{A}^{\prime}, \mathbf{F}, \mathbf{F}^{\prime}\right)$, dpp-RNAi $\left(\mathbf{B}, \mathbf{B}^{\prime}, \mathbf{G}, \mathbf{G}^{\prime}\right)$, sog-RNAi $\left(\mathbf{C}, \mathbf{C}^{\prime}, \mathbf{H}, \mathbf{H}^{\prime}\right)$, tsg-RNAi (D, $\left.\mathbf{D}^{\prime}, \mathbf{I}, \mathbf{I}^{\prime}\right)$ and Toll1-RNAi (E, $\left.\mathbf{E}^{\prime}, \mathbf{J}^{\prime}, \mathbf{J}^{\prime}\right)$ embryos. For each embryo a ventral and a dorsal view, or views from opposite sides if DV polarity is lacking (B-G, D-J) are shown. Magnified surface views to the right of each embryo $\left(x^{\prime}\right)$ reveal the presence or absence of pMAD in individual nuclei. The scale bar $\left(\mathbf{A}, \mathbf{A}^{\prime}\right)$ corresponds to $50 \mu \mathrm{m}$. For identifying the polarity of the DV axis and for BMP signaling activity during later development see Figure 3-figure supplement 1.

DOI: 10.7554/eLife.05502.007

The following figure supplement is available for figure 3 :

Figure supplement 1. Nuclear density and late PMAD distribution identify the dorsal side of Oncopeltus blastoderm embryos.

DOI: 10.7554/eLife.05502.008

result confirms the specificity of our PMAD staining and demonstrates that the KD leads to a severe reduction of BMP signaling in the early embryo (Figure $3 B, G$ ).

Strikingly, this reduction of BMP signaling results in a massive expansion of twi and sog expression around the entire embryonic circumference (Figure 2E,G, for phenotype frequencies see Figure 2-figure supplement 2). The loss of lateral expression of sim (Figure 2F) shows that all fates dorsal to the mesoderm are lacking, indicating that the embryo is completely ventralized. Thus, BMP signaling is required in Oncopeltus to restrict the ventral-most, mesodermal cell fate. Absence of BMP signaling leads to a complete loss of DV polarity, which is not recovered during later stages of development (Figure 4D-F), a phenotype so far not known from other insects where BMP signaling either has no influence on the mesoderm (van der Zee et al., 2006; Lynch and Roth, 2011) or only partially suppresses mesodermal cell fates (Özüak et al., 2014b).

Given the striking expansion of the ventral-most cell fate upon loss of BMP signaling in Oncopeltus, we wondered how ectopic BMP signaling would affect DV patterning. For this purpose we knocked 


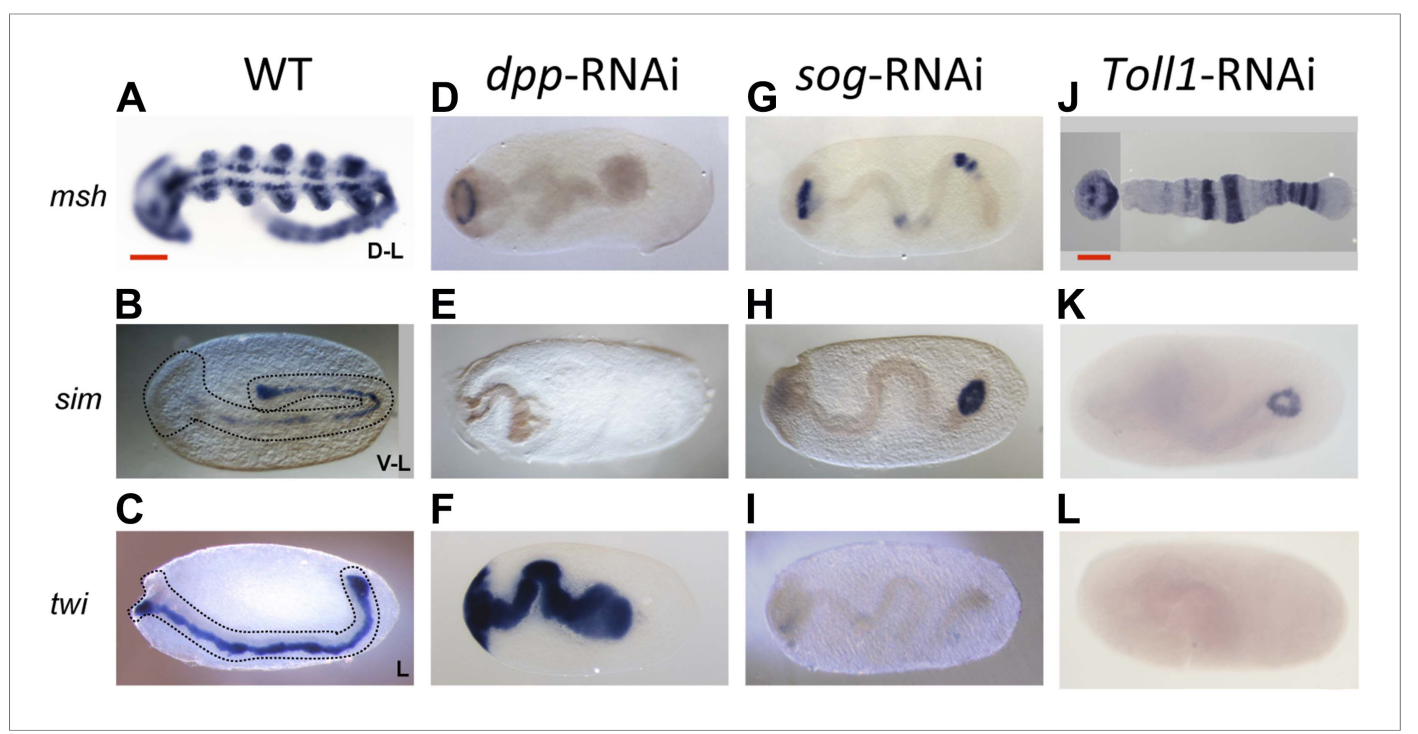

Figure 4. Late phenotypes of dpp, sog and Toll1 KD embryos. Expression of $m s h$ (top row), sim (center row), and twi (bottom row) in wt (A-C), dpp-RNAi (D-F), sog-RNAi (G-I) and Toll1-RNAi (J-L) embryos monitored by ISH. The anterior of the embryo is on the left. Embryos are at the germ band stage ( 40-48 hpf). $\mathbf{m s h}$ : in wt germ band stage embryos msh is expressed in the dorsal-most part of the CNS and in the mesoderm of the limb buds (dorsal-lateral view). dpp-RNAi germ band embryos lack msh expression except for an anterior domain. sog- or Toll1-pRNAi embryos have a tube-like appearance lacking mesoderm and limb buds. Along these tubes msh is either not expressed or it is expressed at uniform levels around the entire circumference. This indicates that the ectoderm of sog- and Toll1 KD embryos is dorsalized either at the level of the dorsal non-neurogenic or the dorsal-most neurogenic ectoderm. sim: in wt germ band stage embryos sim is expressed along the ventral midline (ventrallateral view). Upon dpp-, sog- or Toll1-pRNAi, sim expression is lacking except for a ring of expression at the posterior tip of the growth zone in sog-RNAi and Toll1-RNAi embryos. This indicates that the ventral neuroectoderm is lost in these KD embryos. twi: in germ band stage embryos twi is expressed in the invaginated mesoderm, which forms initially a cord within the embryo (lateral view). In dpp-RNAi embryos twi is expressed in the entire germ band indicating complete mesodermalization. In sog-and Toll1-RNAi embryos twi is not expressed. This, in addition to the loss of sim expression, indicates that sog and Toll1 KD embryos consistently lack ventral cell fates along their entire AP axis. Scale bar corresponds to $200 \mu \mathrm{m}$.

DOI: 10.7554/eLife.05502.009

down sog and twisted gastrulation (tsg). Sog (Chordin) is known from Drosophila and several vertebrates to inhibit BMP signaling via ligand sequestering (Little and Mullins, 2006; O'Connor et al., 2006). For Tsg both anti- and pro-BMP functions have been observed (Wang and Ferguson, 2005; Little and Mullins, 2006; Nunes da Fonseca et al., 2010; Özüak et al., 2014b). In Oncopeltus, the KD of these two genes causes elevated levels of pMAD at the ventral side and frequently leads to a uniform distribution of $\mathrm{PMAD}$ around the embryonic circumference, indicating that the asymmetry of BMP signaling depends on sog and tsg (Figure $3 C, D, H, I)$. The levels of pMAD around the entire circumference are similar to the levels found at the dorsal side of wild type (wt) embryos. The KD embryos show a complete loss of the mesoderm and mesectoderm, as demonstrated by the loss or strong reduction of twi, sog and sim expression (Figure 2I-K,M-O; for phenotype frequencies see Figure 2-figure supplement 2). This further indicates dorsalization and a lack of DV polarity when BMP signaling is uninhibited.

During later development, DV polarity is not recovered: gastrulation and all subsequent morphogenetic movements lack DV asymmetry (Figure 4G-I). Thus, in Oncopeltus, in contrast to all other insects analyzed so far (Lynch and Roth, 2011), BMP signaling has to be suppressed ventrally by Sog (in conjunction with Tsg) to allow polarization of the DV axis and specification of ventral cells. The essential role of Sog is supported by the consequences of a depletion of Tolloid (Tld), which is known to cleave and inactivate Sog and thereby to release bound BMP ligands (O'Connor et al., 2006). As with the $d p p K D$, tld KD leads to a complete ventralization of the embryo, indicating that BMP ligands are largely (or completely) sequestered in inhibitory Sog-BMP complexes in the absence of Tld (Figure 20-S). 
Besides the complete loss of embryonic DV polarity, the KD phenotypes reveal an interesting regulatory feature of the BMP network in Oncopeltus. sog expression is expanded or suppressed by reducing (dpp KD) or expanding (tsg KD) BMP activity, respectively, demonstrating that BMP signaling negatively regulates its own antagonist in Oncopeltus. This was never observed in holometabolous insects, where sog expression either exclusively depends on Toll signaling lacking feedback control by BMP (Drosophila and Tribolium) (Jazwinska et al., 1999; van der Zee et al., 2006) or is absent (Nasonia) (Özüak et al., 2014b). However, in spiders (Akiyama-Oda and Oda, 2006), vertebrates (De Robertis and Kuroda, 2004), and sea anemones (Saina et alo, 2009) the sog homolog chordin is directly or indirectly repressed by BMP signaling, indicating that the BMP network of Oncopeltus exhibits a regulatory property that is ancestral for animals.

As all cell fates along the DV axis are affected by BMP signaling, we wondered whether Toll signaling is even required for DV patterning in Oncopeltus. A recent study in another hemipteran, Rhodnius prolixus has provided evidence that Toll signaling plays a role in DV patterning (Berni et al., 2014). In Oncopeltus, KD of the Toll1 ortholog resulted in loss of twi and sim expression (Figure 5E,F) and sog expression was completely lacking in $38 \%$ of the mid and late blastoderm stage embryos (Figure 5G; for phenotype frequencies and confirmation of KD see Figure 5-figure supplement 1). As expected, this leads to high uniform levels of PMAD around the entire embryo circumference (Figure 3E,J). Expression analysis of germ band stage embryos confirms that the Toll1 KD embryos are dorsalized and lack all DV polarity (Figure 4J-L). KD of other downstream components of Toll signaling (Myd88, Pelle, Tube-like kinase) leads to identical phenotypes (data not shown). Two homologs of NF-kB/Dorsal (Of-dl1 and Of-dl2), the transcription factor acting downstream of Toll signaling (Stein and Stevens, 2014), were identified. KD of both caused a loss of ventral gene expression, albeit to varying degrees, indicating at least partially redundant functions (shown for dl1: Figure 5H-J; Figure 5-figure supplement 1). Taken together, interfering with Toll signaling leads to dorsalized phenotypes, which closely resemble those produced by KD of sog and tsg.

However, loss of Toll signaling also has consequences not observed by manipulating the BMP pathway. This becomes apparent by looking at marker genes expressed in head anlagen like musclespecific homeobox (msh). In wt blastoderm embryos $m s h$ is expressed in a stripe with a sharp anterior border and a posterior border positioned at approximately $60 \%$ egg length ( $0 \%$ is the posterior pole). After Toll1 and dl1 KD the posterior msh border is shifted anteriorly, typically to $80 \%$ egg length, and the stripe expands towards the anterior tip of the embryo (Figure 6B,C). This does not occur in dorsalized embryos after tsg KD (Figure 6D). Using other markers, AP shifts have also not been seen in ventralized embryos after $d p p$ and $t / d K D$ (see anterior sim stripe in Figure 2F, $R$ ). We therefore assume that Toll, unlike BMP signaling, is not only dedicated to DV patterning in Oncopeltus, but also contributes to specifying the AP axis. A role for Toll in positioning the embryo along the AP axis has recently been suggested for the hemipteran Rhodnius (Berni et al., 2014).

Since twi and sog are completely dependent on Toll signaling for their activation in all studied holometabolous insects, we hypothesized that the expansion of twi and sog in dpp KD was due to a corresponding expansion of Toll signaling in the absence of BMP-dependent repression. To test this hypothesis, we produced embryos simultaneously lacking Toll and BMP signaling. To our surprise the Toll1 dpp double KD embryos showed uniform twi and sog expression along the embryonic circumference (Figure 5L, $N$; Figure 5-figure supplement 1 ), the same as the dpp single KD (Figure 2E,G). However, in contrast to the single KD of dpp, the double KD embryos also show an expansion and/or shift of the sog, twi and (anterior) sim domains towards the anterior pole (compare Figure $2 \mathrm{E}-\mathrm{G}$ and Figure $5 \mathrm{~L}-\mathrm{N}$ ). This is likely due to the additional role of Toll signaling in anterior patterning and allows for an unambiguous distinction between double and single KD embryos (additional confirmation by RT-PCR, Figure 5-figure supplement 1). Our results suggest that DV patterning genes that require Toll signaling for expression in other insects can be activated in the absence of Toll signaling in Oncopeltus. As these genes are repressed by elevated BMP activity their state of expression seems to be mainly controlled by different levels of BMP signaling. This leads to the crucial question: What then is Toll's role within the DV patterning system of Oncopeltus if Toll is neither strictly required to activate ventral genes nor to prevent their repression?

To address this question we carefully studied the dynamics of sog expression in wt and Toll1 KD embryos. Interestingly, sog transcription is activated ubiquitously in early blastoderm embryos (Figure 5R). Only later is sog expression enhanced at the ventral side, while weak sog expression is still seen dorsally (Figure 5T, V). Finally, during mid-blastoderm (25-28 hpf) the typical sog expression 


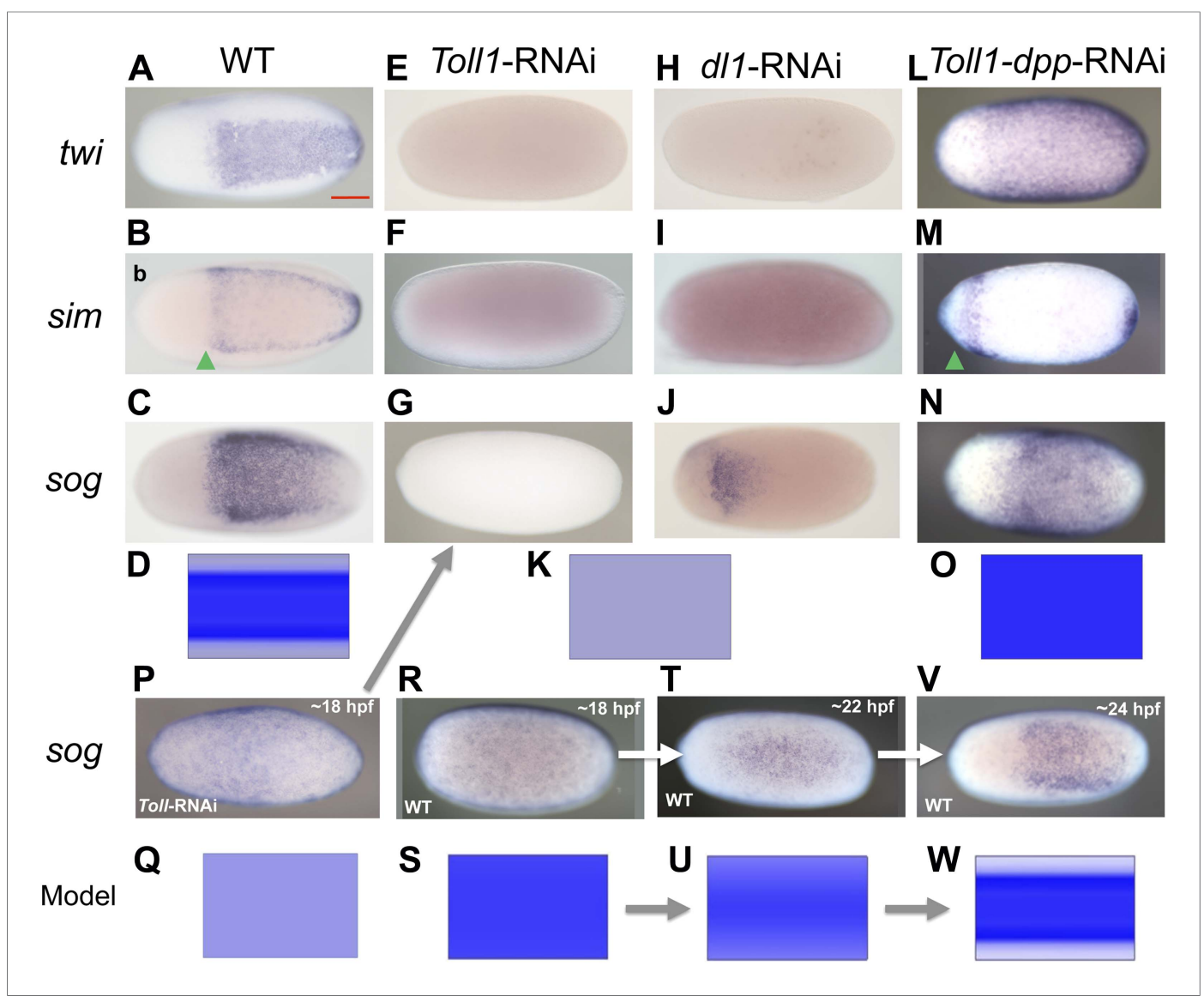

Figure 5. BMP signaling is epistatic to Toll signaling in Oncopeltus. Expression of twi $(\mathbf{A}, \mathbf{E}, \mathbf{H}, \mathbf{L}), \operatorname{sim}(\mathbf{B}, \mathbf{F}, \mathbf{I}, \mathbf{M})$, sog $(\mathbf{C}, \mathbf{G}, \mathbf{J}, \mathbf{N}, \mathbf{P}, \mathbf{R}, \mathbf{T}, \mathbf{V})$ in wt embryos $(\mathbf{A}-\mathbf{C}, \mathbf{R}, \mathbf{T}, \mathbf{V})$, Toll1-RNAi embryos (E-G, P), dl1-RNAi embryos (H-J) and Toll1dpp-RNAi embryos ( $\mathbf{L}-\mathbf{N}$ ) monitored by ISH. The view is ventral $(\mathbf{A}-\mathbf{C}, \mathbf{J}, \mathbf{T}, \mathbf{V})$, or not determined as the expression is DV symmetric (E-G, H, I, L-N, P, R). Embryos are at the blastoderm stage (A-C, E-G, H-J, L-N: $26-32$ hpf; P-V see figure labels). Green arrowheads mark the anterior border of sim expression. The scale bar (A) corresponds to 200 $\mu \mathrm{m}$. For phenotype frequencies and confirmation of $K D$ see Figure 5 -figure supplement 1 . (D, K, O, Q, S, U, W) Simulations of the reaction diffusion system described in Box 1 on a two-dimensional cylinder (Figure 10). Depicted is the ventral part of the cylinder. Blue: sog expression level ( $\eta$ ). Gray: BMP concentration (b). (D) wt: sog expression is confined to a ventral stripe. $(K)$ Upon loss of active NF-KB/Dorsal $(d=0)$ due to either KD of Toll1 or KD of dl1, early activation of $\operatorname{sog}(\mathbf{P})$ is insufficient to initiate patterning resulting in uniformly high BMP signaling. (O) Upon simultaneous loss of Dorsal $(d=0)$ and BMP $(b=0)$ sog activation is possible despite lack of NF-kB/Dorsal; however, activation is uniform. ( $\mathbf{Q})$ sog activation at early stages in the absence of Toll signaling $(d=0)$. This reflects $\eta_{0}, N F-\kappa B /$ Dorsal-independent sog activation (Box 1). (S, U, W) Developmental progression of sog activation $(\eta)$ during blastoderm stages.

DOI: 10.7554/eLife.05502.010

The following figure supplement is available for figure 5 :

Figure supplement 1. Phenotype frequencies and transcript levels after RNAi. DOI: 10.7554/eLife.05502.011

domain is established, with high levels in the ventral $40 \%$ and no detectable expression in the dorsal $60 \%$ of the germ rudiment (Figures $5 \mathrm{C}, 2 \mathrm{C}$ ). Early Toll1 KD embryos show uniform expression of sog (Figure 5P), which disappears during later stages (Figure 5G). Thus, Toll is not required to initiate sog expression, but rather to enhance its expression ventrally. A weakly asymmetric Toll gradient might suffice to fulfill this function.

To get a first impression of the shape of the Toll signaling gradient in Oncopeltus, we analyzed the expression of cactus (cact) genes encoding the insect I-kB homologs which bind to NF- $\mathrm{kB} / D$ orsal and prevent nuclear transport (Bergmann et al., 1996). The transcriptional activation of $I-\kappa B$ genes by Toll 


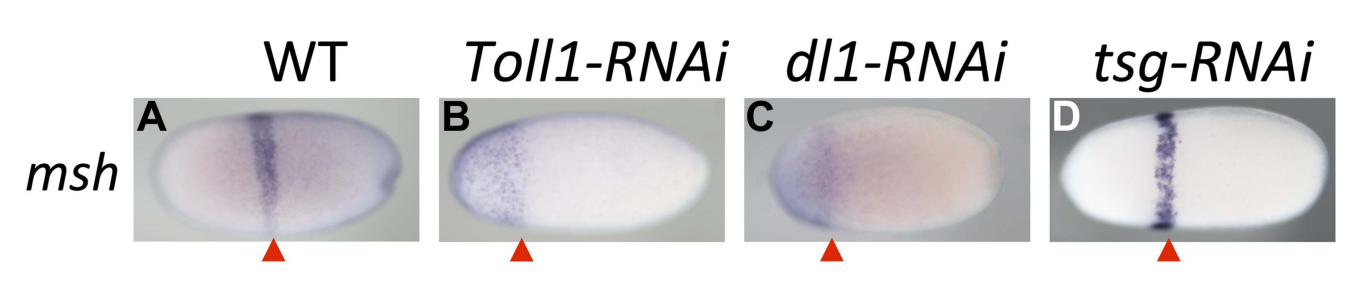

Figure 6. Toll signaling affects AP patterning. Expression of msh is monitored by ISH in blastoderm embryos. The view is lateral (A), or not determined as the expression is DV symmetric (B-D). The red arrowheads mark the posterior border of $m s h$ expression which is positioned at approximately $60 \%$ egg length ( $0 \%$ posterior pole) in wt (A) and tsg KD (D) embryos. In Toll1 and dl1 KD embryos, the msh domain expands to the anterior tip of the embryo and its posterior border is shifted anteriorly (to approximately $80 \%$ egg length).

DOI: 10.7554/eLife.05502.012

signaling appears to be an ancestral negative feedback loop essential for attenuating the innate immune response triggered by Toll (Hoffmann et al., 2002). During Drosophila and Tribolium DV patterning cact is an early target gene of Toll signaling expressed in regions of high nuclear NF-kB/ Dorsal concentrations (Sandmann et al., 2007; Nunes da Fonseca et al., 2008). The same appears to apply to Nasonia where cact expression is restricted to a narrow stripe straddling the ventral midline indicating a highly refined pattern of Toll activity (Buchta et al., 2013; Özüak et al., 2014b). The Oncopeltus genome harbors six cact paralogs, four of which are expressed during blastoderm stages (Vargas Jentzsch et al., 2015) (and data not shown). While cact1, 2 and 4 show only weakly asymmetric expression (Figure 7A-C, and data not shown), cact3 is expressed in a broad ventral domain encompassing $60-80 \%$ of the embryonic circumference with graded borders toward the dorsal side (Figure $7 E-G, I, J)$. The expression of cact3 does not refine into a more narrow domain, but remains broad during later blastoderm stages. Toll1 KD embryos lack (or show reduced) cact1 and cact3 expression, confirming the regulatory link known from other insects (Figure 7D,H). These observations support the notion that in Oncopeltus, Toll signaling is transiently active almost along the entire DV axis and forms a shallow gradient with lower levels in the dorsal half.

In sum, our empirical observations lead to the following model for DV patterning in Oncopeltus (Figure 1, Box 1). We posit that during early blastoderm stages, weak uniform BMP signaling is balanced by the uniform Toll-independent production of the BMP inhibitor Sog. A shallow Toll signaling gradient breaks this symmetry by enhancing sog expression at the ventral side. This leads both to ventral suppression of BMP signaling, and to a flux of BMP-Sog complexes to the dorsal side. Subsequently, the Tld-dependent cleavage of Sog releases BMP and hence increases BMP signaling. Since BMP signaling represses sog expression, the asymmetry initiated by Toll is dynamically enhanced.

To investigate the dynamics of the Sog/BMP system we constructed a minimal reaction-diffusion model as in previous work in Drosophila (Eldar et al., 2002) (Box 1, 'Materials and methods'). In this model the rate of sog expression combines NF-kB/Dorsal-independent and NF-kB/Dorsal-dependent activation with repression of sog by BMP. Parameter settings were selected such that NF-kB/Dorsaldependent sog activation is necessary in order to initiate patterning (Figures 8, 9 and Table 1). This mode of sog activation tightly links DV axis formation to egg polarity via Toll signaling (Stein and Stevens, 2014) and provides stability against random fluctuations (Box 1, 'Materials and methods', Figure 9).

Two-dimensional simulations on a cylinder representing the trunk region of the ellipsoid embryo show that the model robustly replicates the formation of stripe-like sog expression domains (Figure 2D; Figure 5D,W; Figure 10). Moreover, the model correctly recovers the steady state of sog expression and BMP distribution in $K D$ embryos (Figure $2 H, L, P, T$; Figure $5 K, O$ ), including the dynamics of sog expression in Toll1 KD (Figure 5Q, K), as well as wt embryos (Figure 5S, U, W).

Simulations also reveal that even weakly polarized NF-kB/Dorsal gradients result in sharp BMP signaling profiles (Box 1, Figure 9). The final patterning output is robust with regard to variation in width of the NF-kB/Dorsal gradient along the AP axis (Figure 11). Likewise, raising the NF-kB/Dorsal concentration above the critical threshold for sog activation along the entire DV axis had no impact on the patterning output (Figure 9). 


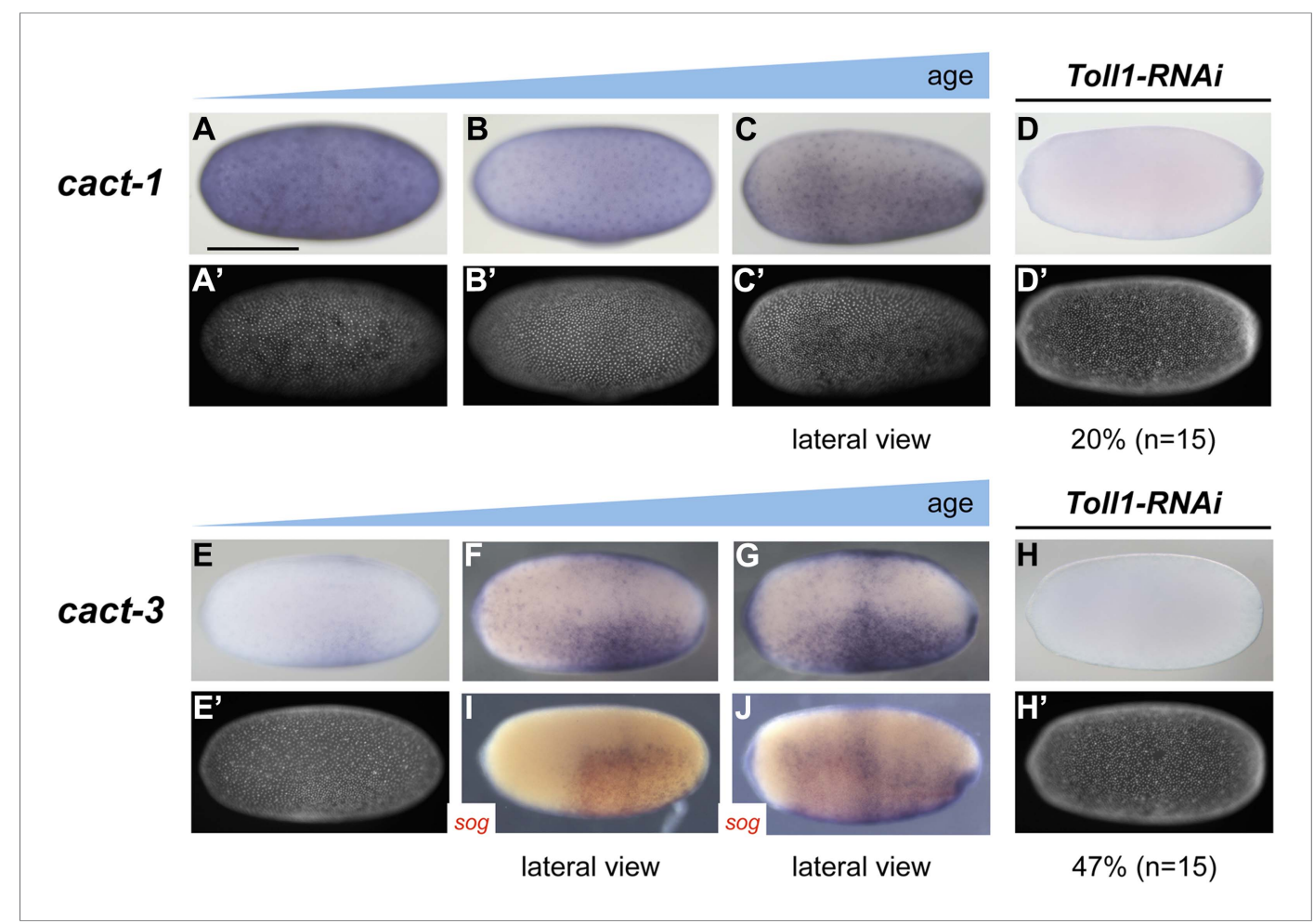

Figure 7. Expression of cact1 and cact3. Expression of cact1 (A-D) and cact3 $(\mathbf{E}-\mathbf{H})$ are monitored by ISH with embryos at early to late blastoderm stages (20-32 hpf). ( $\mathbf{A}^{\prime}-\mathbf{D}^{\prime}, \mathbf{E}^{\prime}, \mathbf{H}^{\prime}$ ) SYTOX Green staining shows nuclear density to determine developmental stage. (A, B) cact1 expression is initiated evenly. (C) With proceeding development cact 1 expression vanishes from the dorsal side. (D) $20 \%$ of Toll1 KD embryos lack cact1 expression. The remainder show reduced expression compared to wt. (E) cact3 expression is initiated uniformly along the DV axis between $20 \%$ and $60 \%$ egg length. $(\mathbf{F}, \mathbf{G})$ In older blastoderm stages cact3 is expressed in a broad domain encompassing 60-80\% of the egg circumference. (H) $47 \%$ of Toll1 KD embryos lack cact3 expression. The remainder show reduced expression compared to wt. (I, J) Double ISH for cact3 (blue) and sog (red) confirms that cact3 is expressed ventrally and that its domain expands more dorsally than the sog domain.

DOI: 10.7554/eLife.05502.013

Such weakly polarized, broad NF-kB/Dorsal distributions in conjunction with the proposed dynamic BMP signaling system can explain embryonic twinning induced by egg fragmentation in another hemipteran species, the leaf hopper Euscelis plebejus (Sander, 1971). In the course of his experiments, Sander produced dorsal and ventral egg fragments during early development (preblastoderm) using a guillotine which completely separated the two egg halves. Complete germ band embryos developed within each half (Box 1). The model proposed here can reproduce such regulative behavior. A split along the DV axis prior to the initiation of patterning prevents diffusion from the ventral to the dorsal half. Thus, BMP acting as a long-range inhibitor of sog expression cannot travel from the ventral to the dorsal half to suppress sog. Since the NF-KB/Dorsal gradient extends to the dorsal half, sog can be activated dorsally and initiates a second round of patterning. Consequently, sog domains and BMP gradients are produced independently in each half despite the NF-kB/Dorsal gradient itself not having been altered (Box 1). The sizes of the sog and BMP domains are adjusted to the dimensions of the egg halves implying that the patterning process shows almost perfect scaling. Furthermore, the predicted orientation of the embryos with ventral sides pointing to the dorsal egg pole (after axis inversion through anatrepsis, [Panfilio, 2008]) corresponds to the most frequently observed experimental outcome (Sander, 1971). Thus, our model represents a minimal BMP/Sog (Chordin) system that exhibits self-organized DV patterning and explains a striking result from classical insect embryology. The only requirement for the NF-kB/Dorsal gradient is that it extends into the dorsal half of the embryo. The expression of cact suggests that this condition is fulfilled in Oncopeltus. Unfortunately, the mechanical properties of Oncopeltus eggs prevents egg fragmentation to directly investigate the potential for twinning. 


\section{Box 1.}

We built a reaction-diffusion model of the BMP/Sog system based on (i) inhibition of sog expression by BMP, (ii) sog transcriptional activation by NF-кB/Dorsal, (iii) the binding of Sog to BMP and (iv) the rapid diffusion of the Sog-BMP complex.

A simple Michaelis-Menten model of sog regulation gives the rate of sog expression

$$
\eta_{\mathrm{s}}(b, d)=\frac{\eta_{0}+\eta_{1} d / d_{0}}{\left(1+b / b_{0}\right)\left(1+d / d_{0}\right)},
$$

in terms of the local concentrations $b(B M P)$ and $d$ (NF-kB/Dorsal). $\eta_{0}$ is the rate of sog expression in the absence of NF- $\mathrm{B}$ /Dorsal and $\mathrm{BMP}, \eta_{1}>\eta_{0}$ is the asymptotic rate of sog expression at high concentrations of NF-кB/Dorsal and in the absence of BMP. This model can exhibit an instability of the homogeneous state (Box figure 1). Consider a small 'seed' of

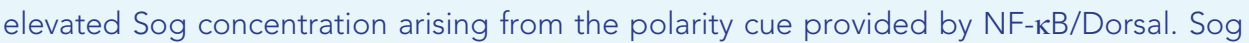
molecules bind BMP and the complexes diffuse away quickly, leading to a depletion of BMP. Since BMP represses sog, this leads to a local increase of sog expression, causing the seed to grow. In the steady state, there is a region of high Sog levels (where BMP diffuses away quickly due to complex formation) around the original seed, and a region of high BMP levels away from the seed, where sog is repressed by BMP.

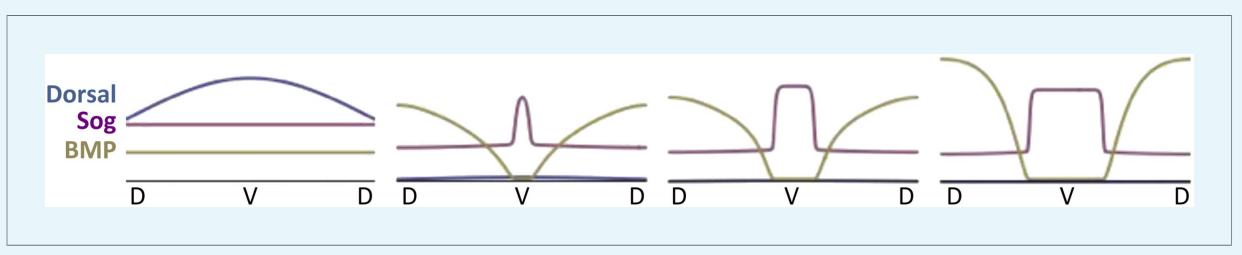

Box figure 1. Temporal progression of pattern formation. Simulated concentration profiles of NF-kB/Dorsal, Sog and BMP are plotted at successive time points, starting from a broad peak of NF-kB/Dorsal (left to right: $\mathrm{t}=0 \mathrm{hr}, 4 \mathrm{hr}, 6 \mathrm{hr}$ and steady state; see Table 1 for parameter values). The $\mathrm{x}$-axis shows the circumference of the embryo, with the dorsal and ventral sides marked as $D$ and $V$, respectively.

DOI: 10.7554/eLife.05502.015

The instability turns out to be controlled by the level of NF-kB/Dorsal. A threshold amount of $\mathrm{NF}-\kappa \mathrm{B} /$ Dorsal is required initially to build a stripe of high Sog concentration near the initial NF$\kappa \mathrm{B} /$ Dorsal maximum (Figures 8, 9). This effect also leads to the phenomenon of twinning: if the amount of NF-kB/Dorsal is above the threshold in both halves of the embryo, a cut along the DV axis can lead to the formation of a stripe in each half (Box figure 2).
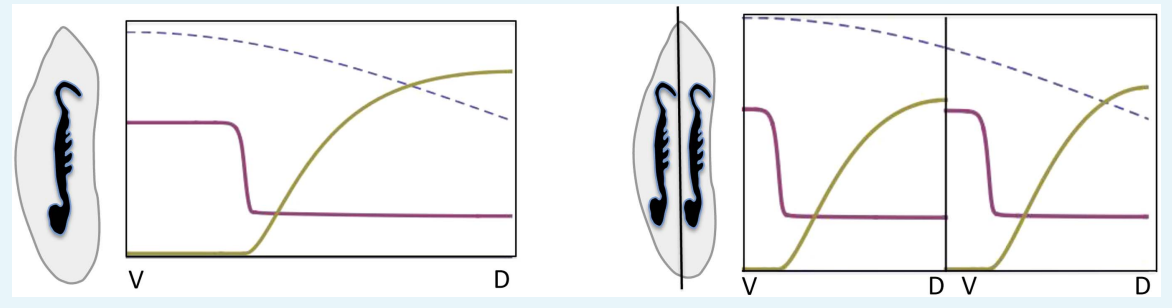

Box figure 2. Embryonic twinning in Euscelis. Simulation of wt (left) showing Sog (red) and BMP (green) protein concentration profiles along the DV axis at steady state given initial NF-kB/Dorsal protein levels (blue: dashed line). A schematic drawing on the left shows a wt Euscelis embryo at the germ band stage. Simulations after the DV axis is split in two halves (right) result in the formation of one BMP gradient in each half. Thus, the proposed model can account for the embryonic twinning observed in Euscelis after production of dorsal and ventral egg fragments shown schematically on the left (Sander, 1971).

DOI: 10.7554/eLife.05502.016 
The dynamics of our model are similar to those of activator-inhibitor models of pattern formation (Turing, 1952; Gierer and Meinhardt, 1972). However, in our model there is no explicit activator. Instead, the patterning mechanism emerges from the transport of the SogBMP complex away from areas with elevated Sog concentration, leading to a derepression of sog by removal of BMP.

DOI: 10.7554/eLife.05502.014

\section{Discussion}

This paper describes three key properties of DV patterning in Oncopeltus. (1) BMP signaling represses the transcription of its extracellular inhibitor sog, a feature so far not observed in other insects, but characteristic for spiders and vertebrates. (2) BMP signaling patterns the entire DV axis by acting repressively on ventral cell fates. This is similar to other bilaterian animals but within insects has so far only been seen in the hymenopteran Nasonia, a representative of the most basally branching holometabolous insect order. (3) Toll is neither a long-range morphogen, as in Drosophila and Tribolium, nor a local inducer of particular cell fates (mesoderm and mesectoderm), as in Nasonia. In Oncopeltus, Toll apparently only acts via its control of BMP signaling. In the following, we discuss these three points and their implications for the evolution of DV patterning in insects.

\section{BMP represses sog in Oncopeltus}

In Drosophila and Tribolium, the expression of sog is dependent on Toll signaling (van der Zee et al., 2006; Liberman and Stathopoulos, 2009; Nunes da Fonseca et al., 2010). sog can be neither activated in absence of Toll (Stathopoulos and Levine, 2004) nor can it be repressed by ectopic BMP signaling (Jazwinska et al., 1999). However, a binding site for transcription factors acting downstream of BMP signaling (Schnurri-Mad-Medea sites) has recently been identified within the proximal enhancer of Drosophila sog (Ozdemir et al., 2014). Its functional significance is not known, but it might represent an evolutionary relict of the regulatory logic we have observed in Oncopeltus in which inhibitory BMP signaling is essential for defining the sog expression domain. A negative feedback of BMP on sog/chordin expression is familiar from many animal phyla. It has even been found in sea anemones, predating the emergence of the bilaterian body plan (Saina et al., 2009;
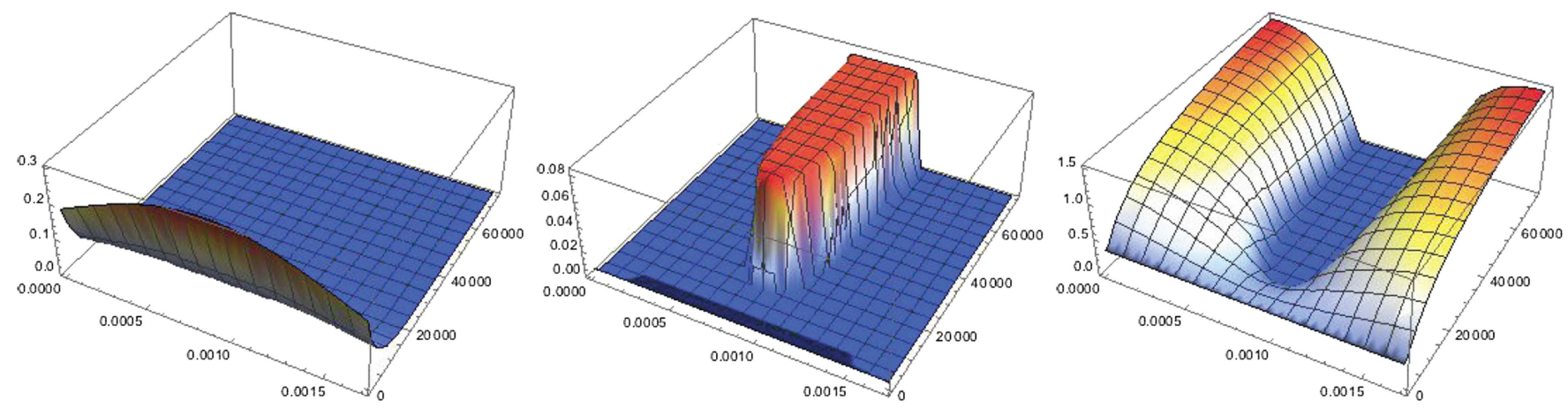

Figure 8. Dynamics of pattern formation. Each plot shows the concentration of a particular protein species in space ( $x$ running from 0 to $I_{x}$ along the front of the plot parameterizing the circumference of the cylinder) and time (running towards the back). Left: the concentration of NF-kB/Dorsal shows a broad Gaussian profile that decays to zero with time. Center: starting from a uniform distribution a region of high Sog concentrations forms where the initial distribution of NF-kB/Dorsal had its maximum. Right: BMP is depleted where Sog levels are high. Initial conditions are $b(x, t=0)=0.32, s(x, t=0)=0.01$, $c(x, t=0)=0.14, d(x, t=0)=D_{o} \exp \left\{-\frac{1}{2}\left(2 / I_{x}\right)^{2}\left(x-2 / I_{x}\right)^{2}\right\}$. Throughout the text $D_{o}=0.3$, except in the twinning figure (Box 1$)$, where $D_{o}=1$ was used to ensure a sufficient amount of NF-kB/Dorsal in both halves of the embryo. 


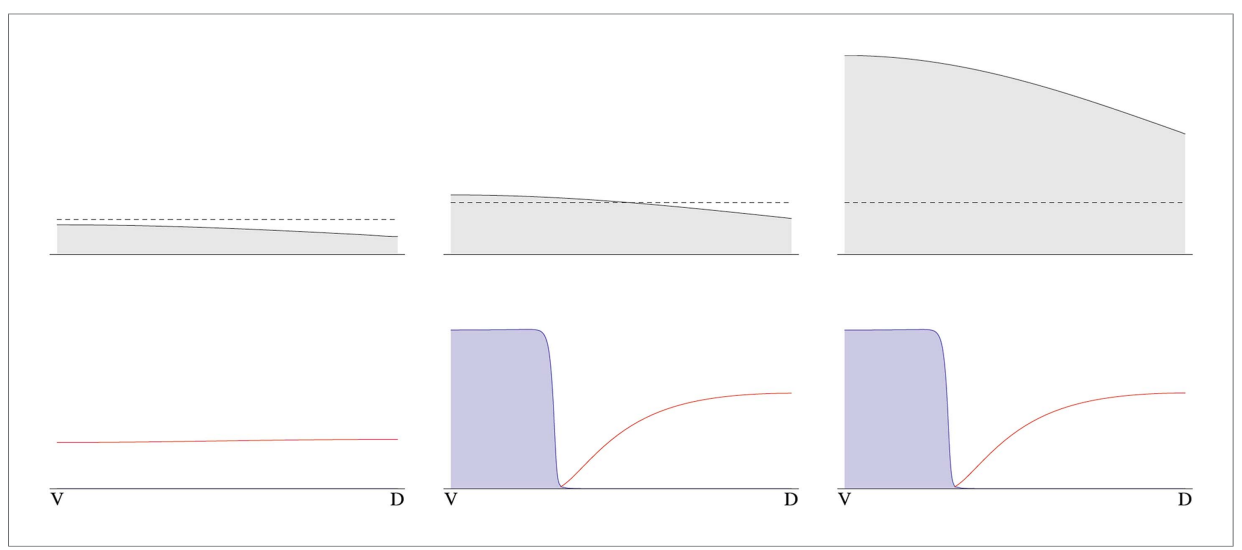

Figure 9. Pattern formation from different initial levels of NF-kB/Dorsal. The initial concentration gradient of NF- $\mathrm{kB} /$ Dorsal is shown on top (gray). Initial amplitudes of NF-kB/Dorsal are $D_{\circ}=0.15,0.3,1$ from left to right, the dashed line indicates the threshold NF-kB/Dorsal concentration required for patterning. Below, steady-state levels of free BMP (red) and free Sog are shown (blue, rescaled to facilitate plotting on the same plot as BMP).

DOI: 10.7554/eLife.05502.018

Genikhovich et al., 2015). For both hemichordates and basal chordates, evidence was provided that BMP suppresses chordin, directly or indirectly (Lowe et al., 2006; Yu et al., 2007). This applies also to spiders, which however have evolved a special strategy of DV axis formation that is radically different from most other animal phyla. In spiders the migration of the BMP expressing cumulus cells towards a symmetric ring of chordin expression breaks DV symmetry (Akiyama-Oda and Oda, 2006). Thus, spiders polarize the DV axis not by localizing inhibitor expression, but rather by localizing BMP.

Among the well-studied DV patterning systems, Oncopeltus can be best compared to vertebrates. In the zebrafish and the frog the negative regulation of chordin by BMP signaling is an important feature to explain normal patterning and axis duplication (twinning) after transplantations (Oelgeschläger et al., 2003; De Robertis, 2009; Langdon and Mullins, 2011; Xue et al., 2014). However, the networks involved in size regulation of the embryonic axis are more complex and require many additional components such as ADMP (antidorsalizing morphogenetic protein), a BMPtype ligand co-expressed with Chordin, and Sizzled, an antagonist of Tld that is co-expressed

Table 1. Model parameters.

\begin{tabular}{ll}
$\eta$ & $2.8 \times 10^{-4}$ \\
\hline$\eta_{1}$ & $2 \times 10^{-3}$ \\
\hline$b_{0}$ & 0.2 \\
\hline$d_{0}$ & 1 \\
\hline$\eta_{b}$ & $4 \times 10^{-5}$ \\
\hline$\alpha_{s}$ & $2 \times 10^{-3}$ \\
\hline$\alpha_{b}$ & $5 \times 10^{-5}$ \\
\hline$\alpha_{c}$ & $2 \times 10^{-4}$ \\
\hline$K_{+}$ & 5 \\
\hline$k_{-}$ & $5 \times 10^{-5}$ \\
\hline$D_{s}$ & $1.5 \times 10^{-13}$ \\
\hline$D_{b}$ & $7.8 \times 10^{-13}$ \\
\hline$D_{c}$ & $2.5 \times 10^{-9}$ \\
\hline$l_{x}$ & 0.0017 \\
\hline
\end{tabular}

Units are arbitrary but are suggested to be seconds for time and meters for length.

DOI: 10.7554/eLife.05502.019 with BMP. ADMP and Sizzled have been implicated in scaling both experimentally and by modeling approaches (Reversade and De Robertis, 2005; Ben-Zvi et al., 2008; Inomata et al., 2013). No homologs of these genes were found in the Oncopeltus genome and transcriptomes (Ewen-Campen et alı, 2011) (Vargas Jentzsch et al., 2015), and appropriate scaling occurred in our theoretical simulations of twinning, without the need to invoke such additional modulators.

Thus, the Oncopeltus system is surprisingly simple and may represent a minimal network able to support self-organized patterning. Our theoretical model emerged from modifications of equations that have been used to describe the formation of peak levels of BMP signaling along the dorsal midline in Drosophila (Eldar et al., 2002). The BMP signaling peak in Drosophila forms within a domain of uniform $d p p$ and t/d expression and depends on both the diffusion of 


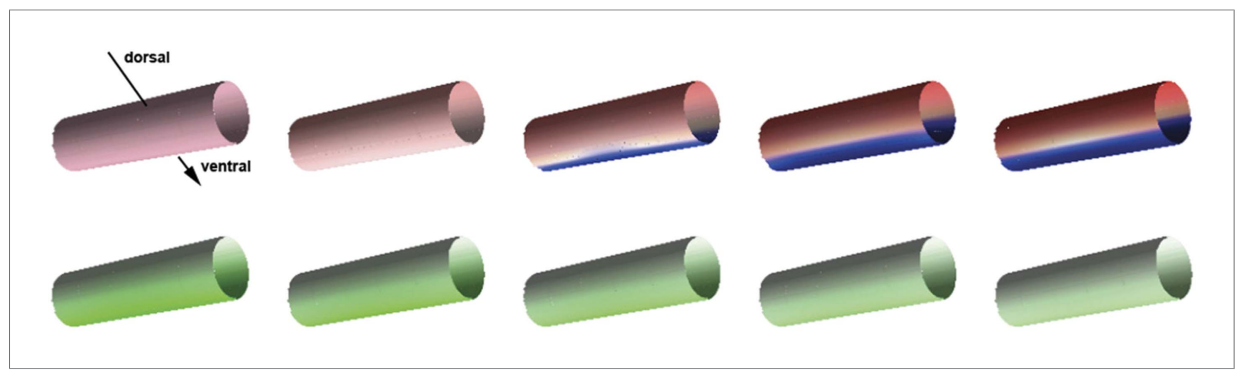

Figure 10. Pattern formation in two dimensions. Starting from a distribution of NF-kB/Dorsal with a broad maximum running in parallel to the cylinder's axis (bottom, shown in green), a stripe of high Sog concentration develops (top, Sog shown in blue, BMP shown in red). The figures show concentrations at times 0, 1000, 2000, 3000, 4000 from left to right.

DOI: 10.7554/eLife.05502.020

Sog-BMP complexes towards the dorsal side (from the ventral source region of sog) and on the degradation of Sog by Tld to release active BMP ligands dorsally. The Drosophila system is static, that is, the regions of ventral sog expression and the abutting regions of $d p p$ and $t / d$ are fixed by the NF$\mathrm{\kappa B} /$ Dorsal gradient. The early dynamics of the system are restricted to reaction diffusion processes. In Oncopeltus, on the other hand, the transcriptional feedback of BMP signaling on sog expression creates a situation in which the size of the sog expression domain itself is an outcome of the system dynamics and becomes largely independent from NF-kB/Dorsal.

In Drosophila and Tribolium the transport of BMP by Sog leads to dorsal BMP signaling levels that are higher than the signaling levels in the absence of Sog (Dorfman and Shilo, 2001; van der Zee et al., 2006). This seems not to be the case in Oncopeltus, as the uniformly high BMP levels seen in sog or tsg KD embryos match the levels found on the dorsal side of control embryos (Figure 3). Furthermore, the BMP profile during normal development is flat, with a narrow and steep transition between uniform BMP signaling dorsally and the absence of BMP signaling ventrally. In our simulations the sog and BMP profiles are also flat, indicating that the negative feedback of BMP
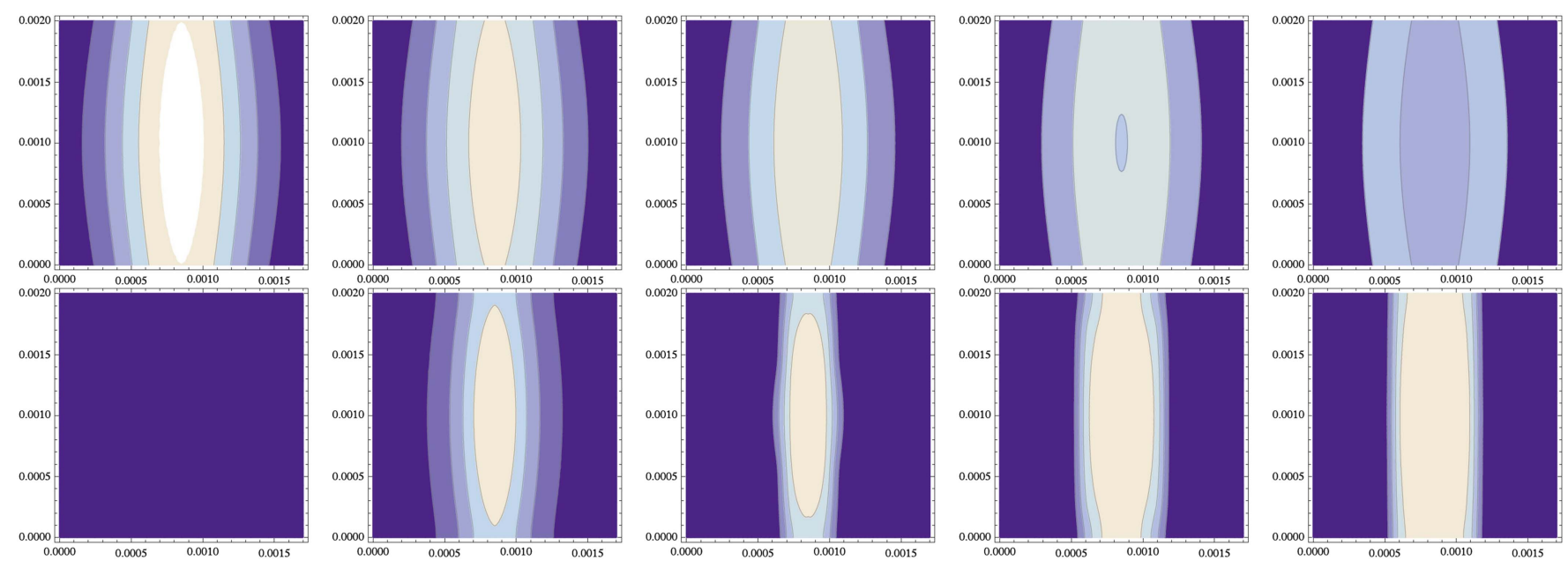

Figure 11. Independence from the stripe of initial conditions (same data as Figure 10). (top) The initial distribution of NF-kB/Dorsal from Figure 10 varies along the cylinder's axis (y-direction of this contour plot, with the $\mathrm{x}$-direction describing the circumference) in both standard deviation and amplitude by about 10\%; $d(x, t=0)=\exp \left\{-\frac{5^{2}}{2\left(1+0.1 \sin \left(\pi y / l_{y}\right)\right)^{2}}\left(x-2 / I_{x}\right)^{2}\right\}\left(1+0.1 \sin \left(\pi y / I_{y}\right)\right)$, and decays over time (time points 0, 1000, 2000, 3000, 4000 shown from left to right). (bottom) The resulting distribution of Sog (and correspondingly of BMP) becomes uniform along the cylinder axis.

DOI: 10.7554/eLife.05502.021 
signaling on sog expression might prohibit the formation of sharp peak levels of BMP signaling (Box 1, Figures 8, 9). Similarly, in vertebrates Chordin-mediated BMP transport does not markedly enhance BMP signaling levels, as chordin KD does not lead to lateralization, but rather to ventralization of the embryo (Schulte-Merker et al., 1997; Oelgeschläger et al., 2003).

An unusual feature of the Oncopeltus system is the strong anti-BMP function of Tsg. In Drosophila Tsg has a mild pro-BMP function that is independent of Sog (Wang and Ferguson, 2005). In Tribolium, Tsg is essential for all BMP activity in a Sog-independent manner (Nunes da Fonseca et al., 2010). The same holds true for Nasonia, which lacks Sog (Özüak et al., 2014b), suggesting that a Sog-independent pro-BMP function of Tsg might be ancestral for insects. To our surprise we observed the opposite in Oncopeltus, where the strong anti-BMP function of tsg is responsible for the similarity of the sog and tsg KD phenotypes (Figures 2,3). Thus, Sog can exert its inhibitory effect on BMP only in the presence of Tsg. It will be interesting to find out whether this strong anti-BMP function of Tsg has a particular significance in a system where sog is repressed by BMP signaling.

We assume that patterning in Oncopeltus is initiated when NF-KB/Dorsal-dependent enhancement of sog expression at the ventral side exceeds a certain threshold. This conclusion is based on the analysis of embryos with incomplete KD of sog (Figures $2 K, 5 \mathrm{~J}$ ). Such embryos frequently show an asymmetric PMAD distribution although they lack ventral gene (twi, sim) expression (Figure 2-figure supplement 2). Thus, ventral BMP signaling has to decrease below a certain threshold to enable normal DV pattern formation. This may provide the system with robustness against fluctuating BMP signaling levels. In our theoretical analysis, the homogeneous steady state of Sog and BMP undergoes a Turing instability when NF-kB/Dorsal-dependent activation of sog reaches a threshold (Figure 9). Then, rapid diffusion of BMP-Sog complexes and derepression of sog upon removal of BMP from the ventral side lead to the formation of a stripe with high expression of sog. The position of the stripe is determined by the initial NF-kB/Dorsal polarity cue.

\section{BMP patterns the entire DV axis in Oncopeltus}

In all insects studied so far the specification of mesodermal and mesectodermal cell fates requires Toll signaling. In Drosophila and Tribolium, the shape of the NF-kB/Dorsal gradient directly or indirectly determines the width of the mesodermal domain (Chen et alı, 2000; Hong et alo, 2008; Nunes da Fonseca et al., 2008). In Nasonia all ventrally expressed genes (e.g., twi) are first turned on in a narrow stripe straddling the ventral midline (Özüak et al., 2014b). This region also shows high levels of cact expression indicating high activity of Toll signaling. Subsequently, cact expression disappears, however, the expression of ventral genes expands. The size of their final domains is determined by repressive BMP signaling from the dorsal side, since KD of BMP leads to progressive expansion of ventral gene expression resulting in a massive, albeit nonuniform expansion of the mesoderm. Thus, in Nasonia BMP effects all subdivisions of the DV axis (Özüak et al., 2014b). However, the expansion of ventral genes remains dependent on their prior activation by Toll, as Toll bmp double KD embryos lack the expression of ventral genes like twi.

In Oncopeltus dpp KD embryos, twi is completely derepressed, that is, its expression is uniform around the embryonic circumference and the developing embryos are fully mesodermalized (Figures 2E, 4F). This phenotype does not result from a progressive expansion of a narrow domain as in Nasonia. Most importantly, the same phenotype is observed in Toll dpp double KD embryos, with the exception that twi expression in addition expands anteriorly due to an AP function of Toll signaling (Figure 5L). These data suggest that Toll signaling in Oncopeltus is no longer strictly required to activate ventral genes. As a consequence, all cell fate decisions along the DV axis of Oncopeltus ultimately depend on different levels of BMP signaling.

As pointed out previously, the early pattern of BMP signaling in Oncopeltus seems to be very simple with a plateau of high signaling at the dorsal side and a broad domain lacking BMP signaling ventrally (Figure 3-figure supplement 1). Accordingly, the early DV fate map of Oncopeltus has apparently only few subdivisions. Although we have thus far not identified genes expressed specifically on the dorsal side, we expect such genes to have broad expression domains encompassing $30-50 \%$ of the embryonic circumference. Likewise, the ventral twi and early sim domains are broad (data not shown). sim refines to lateral stripes bordering twi during later blastoderm stages. None of the columnar genes ( $v n d$, ind, $m s h$ ) show stripe-like expression in lateral regions of the blastoderm as in holometabolous insects (von Ohlen and Doe, 2000; Wheeler et al., 2005; Buchta et al., 2013). We therefore expect that the early BMP gradient provides little patterning 
information on either the dorsal or ventral side, and that further refinement occurs progressively during and after gastrulation. This refinement also includes large-scale morphogenetic movements. For example, the narrow twi domain of germ band embryos (Figure $4 C$ ) results from massive convergent extension during anatrepsis (posterior invagination of the embryo and the amnion into the yolk [Panfilio, 2008]).

\section{In Oncopeltus Toll function is restricted to polarizing BMP signaling}

Several lines of evidence suggest that the Toll gradient acts differently in Oncopeltus compared to the known holometabolous insects: (i) BMP is epistatic to Toll, (ii) ventrally expressed genes can be readily suppressed by increased BMP signaling, (iii) the repression of sog by BMP makes the distribution of BMP signaling largely independent from that of Toll signaling. Although we have not demonstrated the latter point experimentally, modeling shows that adding the repression of sog by BMP to the well known reaction diffusion system (Eldar et al., 2002) leads to a decoupling of input (Toll signaling) and output (BMP signaling) patterns (Figures 9, 11). Toll remains essential at the ventral side to initiate patterning, and therefore, it would be highly interesting to monitor Toll activity by looking at the NF-kB/Dorsal distribution.

In absence of functional antibodies we used the expression of the early Toll target gene cact as a proxy for Toll signaling. In all known holometabolous insects cact is activated by the NF-kB/Dorsal gradient. This applies even to Drosophila as demonstrated with the help of an enhancer reporter construct which shows twist-like expression (Sandmann et alo, 2007). However, due to maternal loading of cact, its zygotic expression apparently has little functional relevance in Drosophila (Roth et al., 1991). In Tribolium, cact is only zygotically expressed and tightly follows the shifting gradient of NF-kB/Dorsal (Nunes da Fonseca et al., 2008). Finally, in Nasonia, cact expression is restricted to a narrow stripe straddling the ventral midline where the expression of all other Toll signalingdependent ventral genes is initiated (Özüak et al., 2014b). Although the NF-KB/Dorsal distribution is not known from Nasonia, a cluster of NF-kB/Dorsal binding sites in the vicinity of the cact transcript suggests a direct regulatory input by Toll signaling. By analogy we assume that the broad, weakly graded expression of cact in Oncopeltus reflects a flat Toll signaling gradient which extends from the ventral to the dorsal half of the embryo (Figure 7 ).

The upstream cascade, which leads to Toll activation in Drosophila (Stein and Stevens, 2014) appears to be largely conserved in Oncopeltus (data not shown). Preliminary data suggest that the asymmetry of Toll signaling originates from asymmetric eggshell cues that are established during oogenesis. By activating Toll signaling in a broad gradient, these eggshell cues would provide global polarity to the embryo, which is essential for establishing bilateral symmetry. Such a strong geometric influence does not exclude, but rather enables, certain forms of self-organized patterning. Classical insect embryology had described many instances of partial or complete twinning after experimental interference with patterning along the DV axis of the egg (Sander, 1976). These experiments were not restricted to hemimetabolous insects, but included examples from beetles as well as butterflies.

The most famous set of experiments was conducted by Sander with Euscelis, a leaf hopper which (like Oncopeltus) belongs to the Hemiptera (Sander, 1971). Sander produced not only left and right, but also dorsal and ventral egg fragments and was able to recover apparently complete germ rudiments from all fragments. These findings could not previously be explained on the basis of the fairly deterministic mechanism of DV axis formation known from Drosophila. The mechanism we have discovered in Oncopeltus can, in principle, account for this type of axis duplication (Box 1). As long as Toll activity is globally provided so that sog can also be activated in the dorsal half of the egg, a new round of patterning can be initiated dorsally. A prerequisite for pattern re-initiation is a diffusion barrier, which prevents the transport of (inhibitory) BMP molecules from the ventral to the dorsal side. The guillotine-like mechanism with which Sander separated the egg fragments provided such a barrier.

In summary, the data presented here not only provide a potential explanation for experiments from classical insect embryology, they also suggest a scenario of how the elaborate morphogen function of Toll signaling found in Drosophila could have originated and evolved (Figure 1). Ancestrally, Toll signaling might have only provided a polarizing function for a self-organizing BMP system responsible for patterning the entire DV axis. Within certain lineages (e.g., flies, beetles and wasps) Toll signaling became more important in directly specifying cell fates along the axis, gradually replacing ancestral BMP functions. 
Our data might also help to explain the transition from the ancestral immune function of Toll found in most metazoan lineages (Gilmore and Wolenski, 2012) to its unique role in DV patterning restricted to insects. Recent findings show that insect eggs are immune competent (Jacobs and van der Zee, 2013; Jacobs et al., 2014). We suggest that this also applied to ancestral insects and that furnishing their eggs with a Toll-mediated pathogen defense system was crucial for early insects to adopt a terrestrial life style. The activation of Toll signaling by eggshell cues might have been fairly uniform throughout the embryo. Subsequently, only a mild polarization of Toll signaling together with weak transcriptional inputs on sog were sufficient to initiate the co-option of Toll signaling for DV patterning.

\section{Materials and methods}

\section{Gene annotation and analysis}

Putative $O$. fasciatus homologs of Toll and BMP signaling components were found by a local blast against a maternal and embryonic transcriptome (Ewen-Campen et al., 2011) using the NCBI blast+ toolkit and BioEdit software, or by degenerate PCR followed by RACE PCR using the SMARTER RACE kit (Clontech, France) to extend the sequence information. Specific primers of all candidates were designed for sequencing, cloning and to confirm the homology with Drosophila and Tribolium (Supplementary file 1). Phylogenetic and molecular evolutionary analyses were then conducted using MEGA version 5 (Tamura et al., 2011) or phylogeny.fr (Dereeper et al., 2008). All Oncopeltus gene sequences have been submitted to GenBank.

\section{RNA interference and KD efficiency validation}

To knock down gene function, gene-specific double-stranded RNA (dsRNA) $(0.1-8 \mu \mathrm{g} / \mu \mathrm{l})$ for parental RNAi was prepared as previously described and injected into virgin females (Nunes da Fonseca et al., 2008). After injection, embryos were collected, fixed and stored in methanol at $-20^{\circ} \mathrm{C}$ as previously described (Liu and Kaufman, 2004) for further phenotype analysis. Total RNA from a single cohort of staged embryos was homogenized and extracted by TRIzol reagent (Life Technologies, Germany) with DNase treatment, and cDNA was synthesized with the VILO Kit (Invitrogen, Germany), following manufacturers' protocols. Gene expression analysis using semi-quantitative RT-PCR was performed using gene-specific primers, with an annealing temperature of $60^{\circ} \mathrm{C}$, and 30 thermocycles.

\section{In situ hybridization and immunohistochemistry}

Detection of gene expression was performed by in situ hybridization (ISH) with digoxigenin-labeled probes as previously described (Liu and Kaufman, 2004). The double ISH was performed with digoxigenin (DIG) and biotin labeled probes hybridized simultaneously followed by incubation with antiBiotin-AP (1:5000, Roche, Germany). After the first round of staining the anti-Biotin-AP antibody was inactivated by treating the embryos with $0.1 \mathrm{M}$ Glycine- $\mathrm{HCl}, \mathrm{pH}=2.2,0.1 \%$ Tween20 for $10 \mathrm{~min}$, followed by washing, blocking and incubation with the second AP antibody (anti-DIG-AP, 1:5000, Roche, Germany). For color reactions we used the HNPP Fluorescent Detection Set (Roche, Germany) and NBT/BCIP. Immunostaining was performed using anti-Phospho-Smad1/5 (41D10) rabbit antibodies (Cell Signaling, Germany) with 1:30 dilution. We introduced the TSA plus DNP system (Perkin Elmer, Waltham, MA) to amplify the signal before DAB detection (or DAB with nickel ammonium sulfate).

\section{Reaction-diffusion model of the BMP/Sog system}

Qualitative description of the model

We build a reaction-diffusion model of the BMP/Sog system in Oncopeltus based on a minimal number of components and their interactions. At the core are the following processes:

- Expression of BMP and of sog. The expression of sog is repressed by BMP and (indirectly) enhanced by NF-kB/Dorsal, whereas BMP is constitutively expressed.

- Binding of Sog protein to BMP protein to form a Sog-BMP complex, and the dissociation of the SogBMP complex.

- Diffusion of BMP, Sog, and the Sog-BMP complex. Crucially, the diffusion constant of the Sog-BMP complex is higher than that of either BMP or Sog.

- The presence of an initial distribution of NF-kB/Dorsal, leading to a locally enhanced expression of sog. This initial distribution acts as a polarity cue.

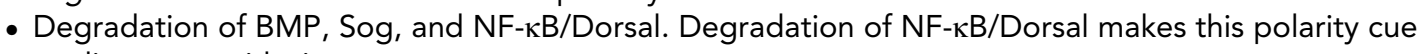
to disappear with time. 
Before formulating and analyzing this model we discuss its dynamics qualitatively. Consider a small 'seed' of elevated Sog concentration in a specific location (for instance as the result of the polarity cue provided by NF-kB/Dorsal). The Sog molecules bind BMP and the complexes diffuse away quickly, leading to a depletion of BMP. Since BMP represses Sog, this leads to a local increase in Sog production, causing the small 'seed' to grow. The steady-state consists of areas where BMP has been depleted (and where levels of Sog are high), bordering areas with high levels of BMP (and low levels of Sog). In the former, sog is not repressed due to the absence of BMP, and BMP is absent due to complex formation with Sog and diffusive transport. In the latter, high levels of BMP repress sog expression. The resulting non-equilibrium steady state will be examined in detail elsewhere.

\section{Comparison with other models}

We consider only one BMP ligand and neglect the fact that BMPs are secreted as homodimers or heterodimers (Shimmi et al., 2005), although we have experimental evidence for a second BMP ligand in Oncopeltus (Figure 2-figure supplement 1). This is in agreement with other recent theoretical models for BMP gradient formation (Mizutani et al., 2005; Shimmi et alo, 2005; Ben-Zvi et al., 2008; Umulis et al., 2010; Peluso et al., 2011; Inomata et al., 2013) and reflects the notion that additional ligands might contribute to increased robustness (Shimmi et al., 2005), but have no essential impact on the mechanisms of pattern formation. The binding of the BMPs to their receptors is not part of our model although we are aware of receptor-mediated degradation affecting the mean free path of the ligand (Mizutani et al., 2005). The model also neglects the binding of Tsg to SogBMP complexes and does not explicitly mention Tolloid (Tld), the enzyme that cleaves Sog. This can be justified by the fact that in blastoderm embryos tsg transcripts are not detectable (by ISH) suggesting very weak uniform expression and that $t / d$ is evenly expressed around the embryonic circumference (Figure 2-figure supplement 1). Similar assumptions have been made in a recent model for BMP signaling in Xenopus (Inomata et al., 2013).

Our minimal model has similarity to one originally suggested by Eldar et al. (2002) (Meinhardt and Roth, 2002) with the crucial difference that sog expression itself is controlled by BMP signaling. The NF-KB/Dorsal gradient in our model plays a similar role as the source density in the GiererMeinhardt model, which was used to explain regulative behavior in hydra (Gierer and Meinhardt, 1972). In fact the mechanism of pattern formation bears some similarity with the local activationlateral inhibition mechanism proposed by Gierer and Meinhardt (1972): BMP inhibits Sog production through transcriptional repression. However, this is the sole regulatory interaction in our model. Local self-activation arises from the fast transport of BMP within Sog-BMP complexes, which moves the repressor for sog from regions of high sog expression (increasing sog expression in those regions) to regions of low sog expression (decreasing sog expression there).

\section{Reaction-diffusion model}

To describe the repression of sog by BMP we use a Michaelis-Menten model; the rate of sog expression is proportional to $1 /\left(1+b / b_{0}\right)$ where $b$ is the concentration of BMP and $b_{0}$ is the concentration of BMP reducing the expression of sog by a factor of two. The constant of proportionality depends on the NF- $\mathrm{kB} /$ Dorsal concentration $d$, which enhances sog expression. In the absence of BMP, the sog expression rate $\left(\eta_{0}+\eta_{1} d / d_{0}\right) /\left(1+d / d_{0}\right)$ extrapolates between $\eta_{0}$ at $d=0$ and $\eta_{1}>\eta_{0}$ at high levels of $d . d_{0}$ denotes the concentration where the intermediate transcription rate $\left(\eta_{0}+\eta_{1}\right) / 2$ is reached. Combining the repression of sog by BMP with its enhancement by NF-kB/Dorsal we obtain the transcription rate of sog as a function of the concentrations of BMP and NF-KB/ Dorsal as,

$$
\eta_{s}(b, d)=\frac{\eta_{0}+\eta_{1} d / d_{0}}{\left(1+b / b_{0}\right)\left(1+d / d_{0}\right)} .
$$

This model of expression regulation can also be derived from a thermodynamic model of two transcription factors (a repressor and an activator) independently binding to a regulatory region.

We model the geometry of the embryo as a cylinder with circumference $I_{x}$. When taking all concentrations to be constant along the axis of the cylinder, one obtains an effectively onedimensional model. This assumption will be examined below, where the dynamics on the surface of the cylinder are considered. In the one-dimensional case, the concentrations $s$ of Sog, b of BMP, $c$ of the Sog-BMP complex, and d of the transcription factor NF-kB/Dorsal depend on time and on the 
variable $x \in\left[0, I_{x}\right]$ running along the circumference of the cylinder, with $x=0$ denoting the ventral side and $\mathrm{x}=\mid x / 2$ the dorsal side.

We now formulate a reaction-diffusion model for the concentrations $s(x, t)$ of Sog, $b(x, t)$ of BMP, $c(x, t)$ of the Sog-BMP complex, and $d(x, t)$ of the transcription factor NF-kB/Dorsal. Under the processes described above, these concentrations evolve as,

$$
\begin{gathered}
\partial_{t} s=D_{s} \nabla^{2} s+\eta_{s}(b, d)-k_{+} s b+k_{-} c-\alpha_{s} s \\
\partial_{t} b=D_{b} \nabla^{2} b+\eta_{b}-k_{+} s b+k_{-} c+\alpha_{s} c-\alpha_{b} b \\
\partial_{t} c=D_{c} \nabla^{2} c+k_{+} s b-k_{-} c-\alpha_{s} c \\
\partial_{t} d=-\alpha_{d} d .
\end{gathered}
$$

In one dimension $\nabla^{2}=\partial^{2} / \partial x^{2}, D$ with appropriate subscript denotes the diffusion constants, analogously $\eta$ the rates of gene expression, $\alpha$ the degradation rates, and $k_{+}$and $k_{-}$the binding and unbinding rates of Sog and BMP. Table 1 gives the parameters used here. We neglect degradation of BMP in the complex, although our results do not depend on this. It turns out that many of these parameters can be changed over at least one order of magnitude without affecting pattern formation (Table 2).

Writing the sog expression rate as $\eta_{s}(b, d) \equiv \bar{\eta}_{s}(d) /\left(1+b / b_{0}\right)$ with $\bar{\eta}_{s}(d) \equiv \frac{\eta_{0}+\eta_{1} d / d_{0}}{1+d / d_{0}}$, we see that the $\mathrm{NF}-\mathrm{kB} /$ Dorsal concentration $d$ controls the difference in sog expression between high and low levels of BMP. We will show below that pattern formation crucially depends on (i) a sufficiently high diffusion rate of the Sog-BMP complex and (ii) repression of sog expression by BMP. The homogeneous state (uniform concentrations of BMP, Sog, and the Sog-BMP complex) can be stable at low levels of $\mathrm{NF}-\mathrm{kB} / \mathrm{Dorsal}$, but becomes unstable (via the mechanism above) once a certain critical NF-kB/Dorsal level is reached. Once a stripe has formed, however, it can persist even in the absence of NF-KB/ Dorsal (Figure 8).

We now explore the dynamics of pattern formation in this model starting from different concentrations of the transcription factor NF-kB/Dorsal (shown in gray), resulting in steady-state concentrations of Sog and BMP shown blue and red, respectively. Starting from small levels of $\mathrm{NF}-\mathrm{KB} /$ Dorsal, a steady state with uniformly low level of Sog arises (Figure 9, left). A threshold amount of NF-kB/Dorsal is required initially to form a stripe of high Sog concentration (Figure 9, center). As a result, small fluctuations in the NF-KB/Dorsal concentration thus do not lead to the formation of a stripe. The size of this stripe does not change if the initial amount of NF-KB/Dorsal is increased

(Figure 9, right). This NF-kB/Dorsal-induced instability of the homogeneous state is also behind

Table 2. Range of model parameter values where a single stripe is formed

\begin{tabular}{ll}
$\overline{\eta_{s}}$ & $6 \times 10^{-4}-1.4 \times 10^{-3}$ \\
\hline$b_{0}$ & $0.1-0.5$ \\
\hline$\eta_{b}$ & $8 \times 10^{-6}-10^{-4}$ \\
\hline$\alpha_{s}$ & $1.6 \times 10^{-3}-1.2 \times 10^{-2}$ \\
\hline$\alpha_{b}$ & $0-10^{-4}$ \\
\hline$K_{+}$ & $0.05-200$ \\
\hline$k_{-}$ & $0-0.5$ \\
\hline$D_{s}$ & $0-10^{-9}$ \\
\hline$D_{b}$ & $0-10^{-11}$ \\
\hline$D_{c}$ & $7 \times 10^{-10}-10^{-6}$ \\
\hline
\end{tabular}

Each parameter is varied keeping the other parameters fixed at the values specified in Table 1. One exception is the parameters $\eta_{0}$ and $\eta_{1}$, which affect pattern formation jointly through the parameter $\bar{\eta}_{s}(d) \equiv \frac{\eta_{0}+\eta_{1} d / d_{0}}{1+d / d_{0}}$ which is set to $1.2 \times 10^{-3}$ (except in the first line, where this parameter itself is varied).

DOI: 10.7554/eLife.05502.022 the twinning phenomenon (Box 1): if the level of NF- $\kappa B / D o r s a l$ exceeds the critical threshold everywhere in the system, a stripe of Sog centered on the maximum of NF-kB/Dorsal forms. Elsewhere, sog is repressed by BMP and transported away from the high-Sog stripe via the Sog-BMP complex. If the system is divided into two separate halves, this transport is interrupted, but the NF-kB/Dorsal level is still above the critical threshold everywhere. Now there are two maxima of NF-kB/Dorsal (one in each of the two halves), and a stripe of Sog forms at each of them.

\section{Stability analysis}

We perform a linear stability analysis to determine when a spatially homogeneous steadystate solution is instable against small sinusoidal spatial oscillations. For now, we consider a spa-

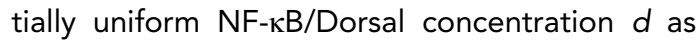
a parameter that can be tuned to take on 
different values. Then the reaction-diffusion Equations (2) become,

$$
\begin{aligned}
& \partial_{t} s=D_{s} \nabla^{2} s+f_{s}(s(x, t), b(x, t), c(x, t)), \\
& \partial_{t} b=D_{b} \nabla^{2} b+f_{b}(s(x, t), b(x, t), c(x, t)), \\
& \partial_{t} c=D_{c} \nabla^{2} c+f_{c}(s(x, t), b(x, t), c(x, t)),
\end{aligned}
$$

with the shorthands,

$$
\begin{gathered}
f_{s}(s, b, c)=\bar{\eta}_{s}(d) /\left(1+b / b_{0}\right)-k_{+} s b+k_{-} c-\alpha_{s} s \\
f_{b}(s, b, c)=\eta_{b}-k_{+} s b+k_{-} c+\alpha_{s} c-\alpha_{b} b \\
f_{c}(s, b, c)=k_{+} s b-k_{-} c-\alpha_{s} c .
\end{gathered}
$$

We now consider a spatially homogeneous fixed point of Equation (3) defined by $s(x, t)=\bar{s}, b(x, t)=\bar{b}, c(x, t)=\bar{c}$ with $0=f_{s}(\bar{s}, \bar{b}, \bar{c})=f_{b}(\bar{s}, \bar{b}, \bar{c})=f_{c}(\bar{s}, \bar{b}, \bar{c})$. In the vicinity of such a fixed point the reaction-diffusion dynamics can be written as,

$$
\partial_{t}\left(\begin{array}{l}
s(x, t) \\
b(x, t) \\
c(x, t)
\end{array}\right)=D \frac{\partial^{2}}{\partial x^{2}}\left(\begin{array}{l}
s(x, t) \\
b(x, t) \\
c(x, t)
\end{array}\right)+A\left(\begin{array}{c}
s(x, t)-\bar{s} \\
b(x, t)-\bar{b} \\
c(x, t)-\bar{c}
\end{array}\right)
$$

where $\mathbf{A}$ is a matrix of partial derivatives evaluated at the fixed point

$$
\mathbf{A}=\left(\begin{array}{lll}
\left.\frac{\partial f_{s}}{\partial s}\right|_{0} & \left.\frac{\partial f_{s}}{\partial b}\right|_{0} & \left.\frac{\partial f_{s}}{\partial c}\right|_{0} \\
\left.\frac{\partial f_{b}}{\partial s}\right|_{0} & \left.\frac{\partial f_{b}}{\partial b}\right|_{0} & \left.\frac{\partial f_{b}}{\partial c}\right|_{0} \\
\left.\frac{\partial f_{c}}{\partial s}\right|_{0} & \left.\frac{\partial f_{c}}{\partial b}\right|_{0} & \left.\frac{\partial f_{c}}{\partial c}\right|_{0}
\end{array}\right)
$$

and $\mathbf{D}$ is the diagonal matrix
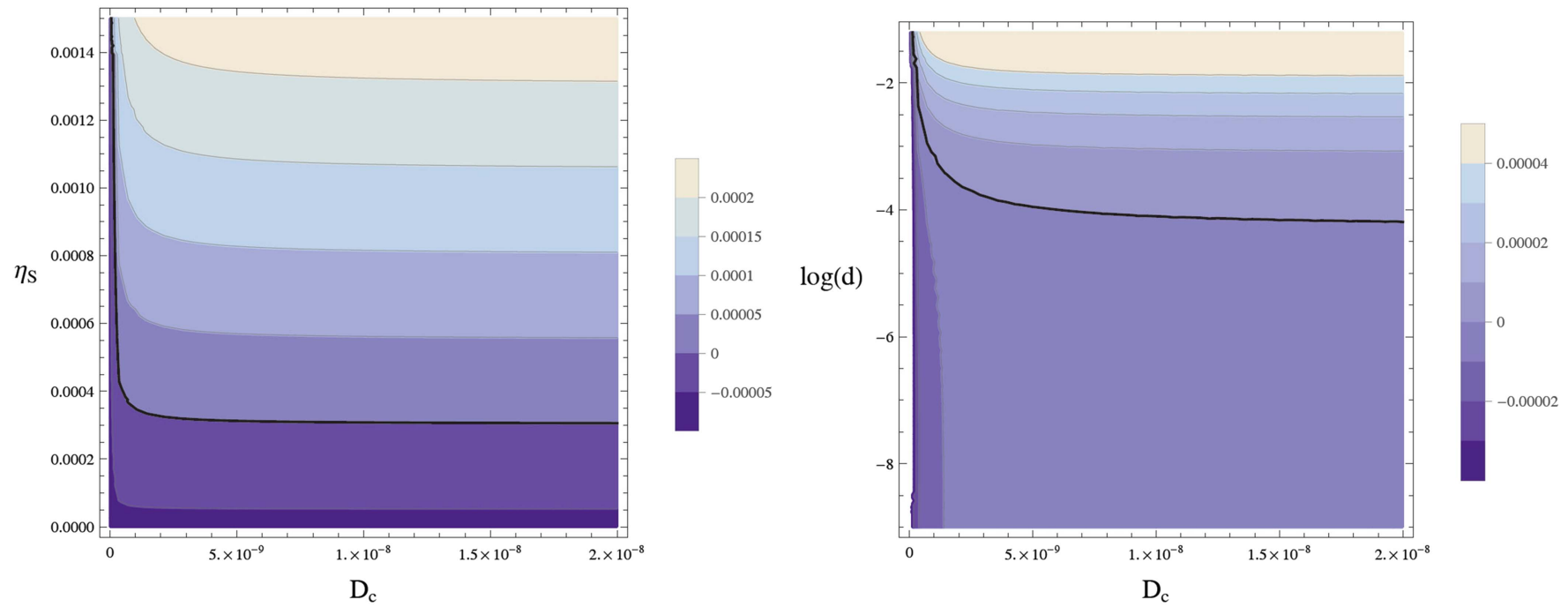

$\mathrm{D}_{\mathrm{c}}$

Figure 12. Stability of the homogeneous fixed point. This contour plot shows the largest eigenvalue $w$ of $\mathbf{A}-\mathbf{D} k^{2}$ for $k=2 \pi / l_{x}$. The thick line separates the parameters leading to a stable homogeneous fixed point $(w<0)$ from an instable homogeneous fixed point ( $w>0)$. (left) $w$ is plotted as a function of the diffusion constant of the Sog-BMP complex and the rate of sog expression at zero BMP, $\bar{\eta}_{s}$. (right) The same data are plotted against log(d) using $\bar{\eta}_{s}(d) \equiv \frac{\eta_{0}+\eta_{1} d / d_{0}}{1+d / d_{0}}$. The homogeneous fixed point becomes unstable for sufficiently large values of the diffusion constant of the complex $D_{c}$ and the concentration of NF-kB/Dorsal $d$. The remaining parameters are as given in Table 1.

DOI: 10.7554/eLife.05502.023 


$$
\mathbf{D}=\left(\begin{array}{lll}
D_{s} & & \\
& D_{s} & \\
& & D_{c}
\end{array}\right) .
$$

The standard ansatz to check stability of the fixed point against small spatial oscillations proceeds by adding a sinusoidal term to the fixed point (Turing, 1952).

$$
\left(\begin{array}{l}
s(x, t) \\
b(x, t) \\
c(x, t)
\end{array}\right)=\left(\begin{array}{l}
\bar{s} \\
\bar{b} \\
\bar{c}
\end{array}\right)+e^{w t+i k x}\left(\begin{array}{l}
\delta s \\
\delta b \\
\delta c
\end{array}\right) .
$$

Inserting this ansatz into Equation (5) gives the eigenvalue equation,

$$
w\left(\begin{array}{c}
\delta s \\
\delta b \\
\delta c
\end{array}\right)=\left(\mathbf{A}-\mathbf{D} k^{2}\right)\left(\begin{array}{l}
\delta s \\
\delta b \\
\delta c
\end{array}\right) .
$$

The fixed point is stable against small spatial sinusoidal perturbations in one spatial dimension if and only if for all values of the wave vector $k$ compatible with the cylindrical geometry all eigenvalues $w$ of the matrix $\mathbf{A}-\mathbf{D} k^{2}$ have a negative real part. Figure 12 shows the largest eigenvalue of $\mathbf{A}-\mathbf{D} k^{2}$ for the wavenumber $k=2 \pi / I_{x}$ that is, the smallest non-zero wavenumber compatible with the circular geometry, showing how the homogeneous steady state becomes unstable for sufficiently large values of $D_{c}$ and $d$.

\section{Dynamics in two dimensions}

We now explore the dynamics of the model constructed above on a two-dimensional cylinder. The surface of the cylinder is described by a variable $y \in\left[0, I_{y}=0.002\right]$ running along its axis, and $x \in\left[0, I_{x}\right]$ running along the circumference. The equations of motion follow from Equation (2) with $\nabla^{2}=\partial^{2} / \partial x^{2}+\partial^{2} / \partial y^{2}$ and open boundary conditions at the two ends of the cylinder. We use the same parameters as in the one-dimensional case (Table 1). Starting from an initial distribution of NF- $\kappa B /$ Dorsal along the ventral side of the cylinder, a stripe of Sog forms along the cylinder (Figure 10). All concentrations turn out to be independent of $y$. We find this also holds in the steady state if the initial distribution of NF-kB/Dorsal varies in the $y$-direction. This becomes more apparent in Figure 11, where the initial distribution of NF-kB/Dorsal from Figure 10 is shown in a contour plot, alongside the steady-state concentration of Sog. Thus, our model produces stripes of constant width (sog expression), which are centered on the ventral midline defined by NF-кB/Dorsal. This is a remarkable feature as it had been difficult to produce striped patterns centered on the midline with local activation-lateral inhibition or substrate depletion mechanisms (Meinhardt, 2004).

\section{Acknowledgements}

This article is dedicated to the memory of the late Klaus Sander (1929-2015), who discovered anteriorposterior morphogen gradients in insects and the regulative patterning along the DV axis. His experiments provided the main motivation for the research conducted in SR's lab, leading to the current paper. We thank Rodrigo Nunes da Fonseca for comments and discussions and are grateful to Waldemar Wojciech and Matt Benton for suggestions, corrections and help with Figure 1. This work has been supported by the CRC 680 of the DFG and the NRW International Graduate School of Development Health and Disease.

\section{Additional information}

Funding

\begin{tabular}{lll} 
Funder & Grant reference & Author \\
\hline Deutsche & CRC 680 & Yen-Ta Chen, Jeremy A \\
Forschungsgemeinschaft & & Lynch, Kristen A Panfilio, \\
(DFG) & & Michael Lässig, Johannes \\
& & Berg, Siegfried Roth \\
\hline
\end{tabular}


Funder

Ministerium für Innovation,

Wissenschaft und

Forschung des Landes

Nordrhein-Westfalen

\section{Grant reference}

NRW International

Graduate School of

Development Health and

Disease

The funders had no role in study design, data collection and interpretation, or the decision to submit the work for publication.

Author contributions

LS, Y-TC, Conception and design, Acquisition of data, Analysis and interpretation of data, Drafting or revising the article; $A D$, Acquisition of data, Analysis and interpretation of data; JAL, KAP, ML, JB, $\mathrm{SR}$, Conception and design, Analysis and interpretation of data, Drafting or revising the article

\section{Additional files}

Supplementary file

- Supplementary file 1. PCR primers for production of ISH probes and dsRNA.

DOI: 10.7554/eLife.05502.024

\section{References}

Akiyama-Oda Y, Oda H. 2006. Axis specification in the spider embryo: dpp is required for radial-to-axial symmetry transformation and sog for ventral patterning. Development 133:2347-2357. doi: 10.1242/dev.02400.

Araujo H, Bier E. 2000. sog and dpp exert opposing maternal functions to modify toll signaling and pattern the dorsoventral axis of the Drosophila embryo. Development 127:3631-3644.

Ben-Zvi D, Shilo BZ, Fainsod A, Barkai N. 2008. Scaling of the BMP activation gradient in Xenopus embryos. Nature 453:1205-1211. doi: 10.1038/nature07059.

Bergmann A, Stein D, Geisler R, Hagenmaier S, Schmid B, Fernandez N, Schnell B, Nüsslein-Volhard C. 1996. A gradient of cytoplasmic Cactus degradation establishes the nuclear localization gradient of the dorsal morphogen in Drosophila. Mechanisms of Development 60:109-123. doi: 10.1016/S0925-4773(96)00607-7.

Berni M, Fontenele MR, Tobias-Santos V, Caceres-Rodrigues A, Mury FB, Vionette-do-Amaral R, Masuda H, Sorgine M, Nunes da Fonseca R, Araujo H. 2014. Toll signals regulate dorsal-ventral patterning and anterior-posterior placement of the embryo in the hemipteran Rhodnius proxilus. EvoDevo 5:38. doi: 10.1186/2041-9139-5-38.

Buchta T, Ozuak O, Stappert D, Roth S, Lynch JA. 2013. Patterning the dorsal-ventral axis of the wasp Nasonia vitripennis. Developmental Biology 381:189-202. doi: 10.1016/j.ydbio.2013.05.026.

Chen G, Handel K, Roth S. 2000. The maternal NF-kappaB/dorsal gradient of Tribolium castaneum: dynamics of early dorsoventral patterning in a short-germ beetle. Development 127:5145-5156.

De Robertis EM. 2008. Evo-devo: variations on ancestral themes. Cell 132:185-195. doi: 10.1016/j.cell.2008.01.003.

De Robertis EM. 2009. Spemann's organizer and the self-regulation of embryonic fields. Mechanisms of Development 126:925-941. doi: 10.1016/j.mod.2009.08.004.

De Robertis EM, Kuroda H. 2004. Dorsal-ventral patterning and neural induction in Xenopus embryos. Annual Review of Cell and Developmental Biology 20:285-308. doi: 10.1146/annurev.cellbio.20.011403.154124.

Dereeper A, Guignon V, Blanc G, Audic S, Buffet S, Chevenet F, Dufayard JF, Guindon S, Lefort V, Lescot M, Claverie JM, Gascuel O. 2008. Phylogeny.fr: robust phylogenetic analysis for the non-specialist. Nucleic Acids Research 36:W465-W469. doi: 10.1093/nar/gkn180.

Dorfman R, Shilo BZ. 2001. Biphasic activation of the BMP pathway patterns the Drosophila embryonic dorsal region. Development 128:965-972.

Eldar A, Dorfman R, Weiss D, Ashe H, Shilo BZ, Barkai N. 2002. Robustness of the BMP morphogen gradient in Drosophila embryonic patterning. Nature 419:304-308. doi: 10.1038/nature01061.

Ewen-Campen B, Shaner N, Panfilio KA, Suzuki Y, Roth S, Extavour CG. 2011. The maternal and early embryonic transcriptome of the milkweed bug Oncopeltus fasciatus. BMC Genomics 12:61. doi: 10.1186/1471-2164-12-61.

Francois V, Solloway M, O'Neill JW, Emery J, Bier E. 1994. Dorsal-ventral patterning of the Drosophila embryo depends on a putative negative growth factor encoded by the short gastrulation gene. Genes \& Development 8: 2602-2616. doi: 10.1101/gad.8.21.2602.

Franzenburg S, Fraune S, Künzel S, Baines JF, Domazet-Loso T, Bosch TC. 2012. MyD88-deficient Hydra reveal an ancient function of TLR signaling in sensing bacterial colonizers. Proceedings of the National Academy of Sciences of USA 109:19374-19379. doi: 10.1073/pnas.1213110109.

Garcia M, Stathopoulos A. 2011. Lateral gene expression in Drosophila early embryos is supported by Grainyheadmediated activation and tiers of dorsally-localized repression. PLOS ONE 6:e29172. doi: 10.1371/journal.pone.0029172.

Genikhovich G, Fried P, Prünster MM, Schinko JB, Gilles AF, Fredman D, Meier K, Iber D, Technau U. 2015. Axis patterning by BMPs: Cnidarian network reveals evolutionary constraints. Cell Reports doi: 10.1016/j.celrep.2015. 02.035 . 
Gierer A, Meinhardt H. 1972. A theory of biological pattern formation. Kybernetik 12:30-39. doi: 10.1007/ BF00289234.

Gilmore TD, Wolenski FS. 2012. NF-kappaB: where did it come from and why? Immunological Reviews 246:14-35. doi: 10.1111/j.1600-065X.2012.01096.x.

Hoffmann A, Levchenko A, Scott ML, Baltimore D. 2002. The IkappaB-NF-kappaB signaling module: temporal control and selective gene activation. Science 298:1241-1245. doi: 10.1126/science.1071914.

Hong JW, Hendrix DA, Papatsenko D, Levine MS. 2008. How the dorsal gradient works: insights from postgenome technologies. Proceedings of the National Academy of Sciences of USA 105:20072-20076. doi: 10.1073/pnas. 0806476105.

Inomata H, Shibata T, Haraguchi T, Sasai Y. 2013. Scaling of dorsal-ventral patterning by embryo size-dependent degradation of Spemann's organizer signals. Cell 153:1296-1311. doi: 10.1016/j.cell.2013.05.004.

Jacobs CG, Spaink HP, van der Zee M. 2014. The extraembryonic serosa is a frontier epithelium providing the insect egg with a full-range innate immune response. eLife 3:e04111. doi: 10.7554/eLife.04111.

Jacobs CG, van der Zee M. 2013. Immune competence in insect eggs depends on the extraembryonic serosa. Developmental and Comparative Immunology 41:263-269. doi: 10.1016/j.dci.2013.05.017.

Jazwinska A, Rushlow C, Roth S. 1999. The role of brinker in mediating the graded response to Dpp in early Drosophila embryos. Development 126:3323-3334.

Langdon YG, Mullins MC. 2011. Maternal and zygotic control of zebrafish dorsoventral axial patterning. Annual Review of Genetics 45:357-377. doi: 10.1146/annurev-genet-110410-132517.

Leulier F, Lemaitre B. 2008. Toll-like receptors-taking an evolutionary approach. Nature Reviews Genetics 9: 165-178. doi: 10.1038/nrg2303.

Liberman LM, Stathopoulos A. 2009. Design flexibility in cis-regulatory control of gene expression: synthetic and comparative evidence. Developmental Biology 327:578-589. doi: 10.1016/j.ydbio.2008.12.020.

Little SC, Mullins MC. 2006. Extracellular modulation of BMP activity in patterning the dorsoventral axis. Birth Defects Research Part C, Embryo Today: Reviews 78:224-242. doi: 10.1002/bdrc.20079.

Liu P, Kaufman TC. 2009. Morphology and husbandry of the large milkweed bug, Oncopeltus fasciatus. Cold Spring Harbor Protocols 2009:pdb emo127. doi: 10.1101/pdb.emo127.

Liu PZ, Kaufman TC. 2004. hunchback is required for suppression of abdominal identity, and for proper germband growth and segmentation in the intermediate germband insect Oncopeltus fasciatus. Development 131: 1515-1527. doi: 10.1242/dev.01046.

Lowe CJ, Terasaki M, Wu M, Freeman RM Jr, Runft L, Kwan K, Haigo S, Aronowicz J, Lander E, Gruber C, Smith M, Kirschner M, Gerhart J. 2006. Dorsoventral patterning in hemichordates: insights into early chordate evolution. PLOS Biology 4:e291. doi: 10.1371/journal.pbio.0040291.

Lynch JA, Roth S. 2011. The evolution of dorsal-ventral patterning mechanisms in insects. Genes \& Development 25:107-118. doi: 10.1101/gad.2010711.

Meinhardt H. 2004. Different strategies for midline formation in bilaterians. Nature Reviews Neuroscience 5: 502-510. doi: 10.1038/nrn1410.

Meinhardt H, Roth S. 2002. Developmental biology: sharp peaks from shallow sources. Nature 419:261-262. doi: 10.1038/419261a.

Mizutani CM, Nie Q, Wan FY, Zhang YT, Vilmos P, Sousa-Neves R, Bier E, Marsh JL, Lander AD. 2005. Formation of the BMP activity gradient in the Drosophila embryo. Developmental Cell 8:915-924. doi: 10.1016/j.devcel.2005. 04.009.

Nunes da Fonseca R, van der Zee M, Roth S. 2010. Evolution of extracellular Dpp modulators in insects: the roles of tolloid and twisted-gastrulation in dorsoventral patterning of the Tribolium embryo. Developmental Biology 345:80-93. doi: 10.1016/j.ydbio.2010.05.019.

Nunes da Fonseca R, von Levetzow C, Kalscheuer P, Basal A, van der Zee M, Roth S. 2008. Self-regulatory circuits in dorsoventral axis formation of the short-germ beetle Tribolium castaneum. Developmental Cell 14:605-615. doi: 10.1016/j.devcel.2008.02.011.

O'Connor MB, Umulis D, Othmer HG, Blair SS. 2006. Shaping BMP morphogen gradients in the Drosophila embryo and pupal wing. Development 133:183-193. doi: 10.1242/dev.02214.

Oelgeschläger M, Kuroda H, Reversade B, De Robertis EM. 2003. Chordin is required for the Spemann organizer transplantation phenomenon in Xenopus embryos. Developmental Cell 4:219-230. doi: 10.1016/S1534-5807(02) 00404-5.

Ozdemir A, Ma L, White KP, Stathopoulos A. 2014. Su(H)-mediated repression positions gene boundaries along the dorsal-ventral axis of Drosophila embryos. Developmental Cell 31:100-113. doi: 10.1016/j.devcel.2014.08.005.

Özüak O, Buchta T, Roth S, Lynch JA. 2014a. Ancient and diverged TGF-beta signaling components in Nasonia vitripennis. Development Genes and Evolution 224:223-233. doi: 10.1007/s00427-014-0481-0.

Özüak O, Buchta T, Roth S, Lynch JA. 2014b. Dorsoventral polarity of the Nasonia embryo primarily relies on a BMP gradient formed without input from Toll. Current Biology 24:2393-2398.

Panfilio KA. 2008. Extraembryonic development in insects and the acrobatics of blastokinesis. Developmental Biology 313:471-491. doi: 10.1016/j.ydbio.2007.11.004.

Panfilio KA, Liu PZ, Akam M, Kaufman TC. 2006. Oncopeltus fasciatus zen is essential for serosal tissue function in katatrepsis. Developmental Biology 292:226-243. doi: 10.1016/j.ydbio.2005.12.028.

Peluso CE, Umulis D, Kim YJ, O'Connor MB, Serpe M. 2011. Shaping BMP morphogen gradients through enzymesubstrate interactions. Developmental Cell 21:375-383. doi: 10.1016/j.devcel.2011.06.025.

Reeves GT, Stathopoulos A. 2009. Graded dorsal and differential gene regulation in the Drosophila embryo. Cold Spring Harbor Perspectives in Biology 1:a000836. doi: 10.1101/cshperspect.a000836. 
Reversade B, De Robertis EM. 2005. Regulation of ADMP and BMP2/4/7 at opposite embryonic poles generates a self-regulating morphogenetic field. Cell 123:1147-1160. doi: 10.1016/j.cell.2005.08.047.

Roth S, Hiromi Y, Godt D, Nüsslein-Volhard C. 1991. cactus, a maternal gene required for proper formation of the dorsoventral morphogen gradient in Drosophila embryos. Development 112:371-388.

Rushlow CA, Shvartsman SY. 2012. Temporal dynamics, spatial range, and transcriptional interpretation of the Dorsal morphogen gradient. Current Opinion in Genetics \& Development 22:542-546. doi: 10.1016/j.gde.2012.08.005.

Saina M, Genikhovich G, Renfer E, Technau U. 2009. BMPs and chordin regulate patterning of the directive axis in a sea anemone. Proceedings of the National Academy of Sciences of USA 106:18592-18597. doi: 10.1073/pnas. 0900151106.

Sander K. 1971. Pattern formation in longitudinal halves of leaf hopper eggs (Homoptera) and some remarks on the definition of 'embryonic regulation'. Wilhelm Roux' Archiv für Entwicklungsmechanik der Organismen 167: 336-352. doi: 10.1007/BF00580168.

Sander K. 1976. Specification of the basic body pattern in insect embryogenesis. Advances in Insect Physiology 12: 125-238. doi: 10.1016/S0065-2806(08)60255-6.

Sandmann T, Girardot C, Brehme M, Tongprasit W, Stolc V, Furlong EE. 2007. A core transcriptional network for early mesoderm development in Drosophila melanogaster. Genes \& Development 21:436-449. doi: 10.1101/ gad.1509007.

Schulte-Merker S, Lee KJ, McMahon AP, Hammerschmidt M. 1997. The zebrafish organizer requires chordino. Nature 387:862-863. doi: 10.1038/43092.

Shimmi O, Umulis D, Othmer H, O'Connor MB. 2005. Facilitated transport of a Dpp/Scw heterodimer by Sog/Tsg leads to robust patterning of the Drosophila blastoderm embryo. Cell 120:873-886. doi: 10.1016/j.cell.2005.02.009.

Stathopoulos A, Levine M. 2004. Whole-genome analysis of Drosophila gastrulation. Current Opinion in Genetics \& Development 14:477-484. doi: 10.1016/j.gde.2004.07.004.

Stein DS, Stevens LM. 2014. Maternal control of the Drosophila dorsal-ventral body axis. Wiley Interdisciplinary Reviews Developmental Biology 3:301-330. doi: 10.1002/wdev.138.

Tamura K, Peterson D, Peterson N, Stecher G, Nei M, Kumar S. 2011. MEGA5: molecular evolutionary genetics analysis using maximum likelihood, evolutionary distance, and maximum parsimony methods. Molecular Biology and Evolution 28:2731-2739. doi: 10.1093/molbev/msr121.

Thisse B, Stoetzel C, Gorostiza-Thisse C, Perrin-Schmitt F. 1988. Sequence of the twist gene and nuclear localization of its protein in endomesodermal cells of early Drosophila embryos. The EMBO Journal 7:2175-2183.

Thomas JB, Crews ST, Goodman CS. 1988. Molecular genetics of the single-minded locus: a gene involved in the development of the Drosophila nervous system. Cell 52:133-141. doi: 10.1016/0092-8674(88)90537-5.

Turing AM. 1952. The chemical basis of morphogenesis. Philosophical Transactions of the Royal Society B: Biological Sciences 237:37-72. doi: 10.1098/rstb.1952.0012.

Umulis DM, Shimmi O, O'Connor MB, Othmer HG. 2010. Organism-scale modeling of early Drosophila patterning via bone morphogenetic proteins. Developmental Cell 18:260-274. doi: 10.1016/j.devcel.2010.01.006.

van der Zee M, Stockhammer O, von Levetzow C, Nunes da Fonseca R, Roth S. 2006. Sog/Chordin is required for ventral-to-dorsal Dpp/BMP transport and head formation in a short germ insect. Proceedings of the National Academy of Sciences of USA 103:16307-16312. doi: 10.1073/pnas.0605154103.

Vargas Jentzsch IM, Hughes DST, Poelchau MFT, The O. fasciatus curation community, Richards S, Panfilio KA. 2015. Oncopeltus fasciatus official gene set v1.1. http://dx.doi.org/10.15482/USDA.ADC/1173142.

von Ohlen T, Doe CQ. 2000. Convergence of dorsal, dpp, and egfr signaling pathways subdivides the Drosophila neuroectoderm into three dorsal-ventral columns. Developmental Biology 224:362-372. doi: 10.1006/dbio.2000.9789.

Wang YC, Ferguson EL. 2005. Spatial bistability of Dpp-receptor interactions during Drosophila dorsal-ventral patterning. Nature 434:229-234. doi: 10.1038/nature03318.

Wheeler SR, Carrico ML, Wilson BA, Skeath JB. 2005. The Tribolium columnar genes reveal conservation and plasticity in neural precursor patterning along the embryonic dorsal-ventral axis. Developmental Biology 279: 491-500. doi: 10.1016/j.ydbio.2004.12.031.

Xue Y, Zheng X, Huang L, Xu P, Ma Y, Min Z, Tao Q, Tao Y, Meng A. 2014. Organizer-derived Bmp2 is required for the formation of a correct Bmp activity gradient during embryonic development. Nature Communications $\mathbf{5}$ : 3766. doi: 10.1038/ncomms4766.

Yu JK, Satou Y, Holland ND, Shin IT, Kohara Y, Satoh N, Bronner-Fraser M, Holland LZ. 2007. Axial patterning in cephalochordates and the evolution of the organizer. Nature 445:613-617. doi: 10.1038/nature05472. 